\title{
Progress in Cellulose/Carbon Nanotube Composite Flexible Electrodes for Supercapacitors
}

\author{
Zhe Sun, ${ }^{1}$ Houjuan Qi, ${ }^{1}$ Manhui Chen, ${ }^{1}$ Sitong Guo, ${ }^{1}$ Zhanhua Huang,,${ }^{*}$ Srihari Maganti, ${ }^{2}$ Vignesh Murugadoss,${ }^{3}$ Mina Huang ${ }^{2,3}$ \\ and Zhanhu Guo ${ }^{2 *}$
}

\begin{abstract}
Nowadays, there is an increasing demand for wearable, portable and foldable small electronic products, and humancomputer interaction interface devices. Therefore, supercapacitors as energy storage devices were extensively studied owing to their high energy/power density, fast charge-discharge processes, and long cycle life. Wherein the flexible electrode material is the essential component to boost the performance of supercapacitors. Cellulose, as a kind of natural flexible material with low-cost, wide-sourced, renewable, and robust mechanical properties, has been used as a flexible substrate or template of electrodes. To enhance the conductivity and excellent electrochemical performance of the cellulose-based flexible electrode, the carbon nanotube (CNT) with high conductivity, good thermal and chemical stability, and a unique internal structure was integrated. Thereby, the cellulose/CNT-based flexible electrodes with high energy/power density and long cycle life performance of flexible supercapacitors are prepared. This review mainly focuses on cellulose/CNT, emphatically summarizing the composition, preparation, and mechanism of the cellulose/CNT-based composite flexible electrode for supercapacitors. Additionally, the current challenges and prospects of the cellulose/CNT-based composite flexible electrode are discussed.
\end{abstract}

Keywords: Cellulose; Nanocellulose; Carbon nanotube; Supercapacitor; Flexible electrode.

Received: 11 October 2021; 02 December 2021; Accepted: 03 December 2021.

Article type: Review article.

\section{Introduction}

With the substantial increase in market demand for highperformance, wearable, portable and foldable electronic devices, the flexible supercapacitor with high energy-power density, long cycle life, and fast charge-discharge speed has aroused great interest. As new energy storage devices, flexible supercapacitors were comprehensive research in the field of electronics, electric vehicles, intelligent networks, defense, and aerospace. ${ }^{[1-7]}$ The flexible electrode, as a key component

\footnotetext{
${ }^{1}$ Key Laboratory of Bio-based Materil Science and Technology, Ministry of Education, College of Material Science and Engineering, Northeast Forestry University, Harbin, 150040, China.

${ }^{2}$ Integrated Composites Laboratory (ICL), Department of Chemical and Bimolecular Engineering, University of Tennessee, Knoxville, TN, 37996, United States.

3 Advanced Materials Division, Engineered Multifunctional Composites (EMC) Nanotech LLC, Knoxville, TN 37934, United States.

*Email: huangzh1975@163.com (Z. Huang),

nanomaterials2000@gmail.com (Z.Guo)
}

of flexible supercapacitors, will directly affect the electrochemical performance of the devices, such as specific capacitance, energy-power density, and cycle stability. However, flexible electrodes still have small capacitance, poor conductivity, and low energy density in energy storage devices. Therefore, flexible electrode materials with lightweight and excellent electrochemical performance need to be selected, developed, and designed. This is one of the main development directions of flexible device energy storage in the future.

Cellulose, a natural biological macromolecule with low cost, abundant sources, biodegradability, easy processing, and excellent mechanical properties, is often studied as a matrix or template for flexible electrode materials. According to different raw materials, cellulose fibril size, and pretreatment methods, the treated cellulose can be divided into nanocellulose and cellulose microfiber. ${ }^{[8]}$ The pretreated cellulose has high mechanical strength, large specific surface area, high Young's modulus, strong hydrophilicity, high aspect ratio, excellent mechanical properties, good chemical and thermal stability, high porosity, and easy dispersion. ${ }^{[9-16]}$ Considering the advantages of cellulose, it has attracted extraordinary 
attention in the fields of flexible electrochemical energy storage devices (such as high energy and power density electrodes, current collectors, and functional separators). ${ }^{[17]}$

As shown in Fig. 1a, the Ragone diagram summarizes the relationship between the energy density and power density of cellulose composite electrode materials from different sources in supercapacitors. ${ }^{[18-32]}$ This result provides the theoretical basis for the subsequent accurate and effective composite with conductive materials to improve their electrochemical performance. Although the cellulosic composite electrode material showed higher energy density and power density in the Ragone diagram, there is still enormous space for development and improvement.

To solve the problem that cellulose itself is nonconductivity, many studies have combined it with conductiveactive materials to form cellulose-based composites. Currently, the commonly used conductive active materials include carbon nanotubes (CNT), redox graphene (rGO), conductive polymers (e.g. polyaniline (PANI), polypyrrole (PPy), and polythiophene (PEDOT)), and metals (such as metal oxide and metal hydroxide). ${ }^{[33-39]}$ Recently, CNT has been well investigated in the electrochemical energy storage field because of its high conductivity, large surface area, well contact with the electrolyte, good chemical and thermal stability, excellent mechanical properties, and easy functionalization. The flexible electrode materials for supercapacitors are prepared by combining them with cellulose via the interaction of hydrogen bonds and van der Waals forces and have high conductivity, eco-friendly, and excellent mechanical properties. ${ }^{[40-43]}$ Fig. $1 \mathrm{~b}$ concludes the electrochemical performance of different cellulose/CNTbased composite flexible electrodes in supercapacitors. ${ }^{[31,44-47]}$

In the cellulose/CNT composite material, cellulose can be used as a dispersant and surfactant to improve the
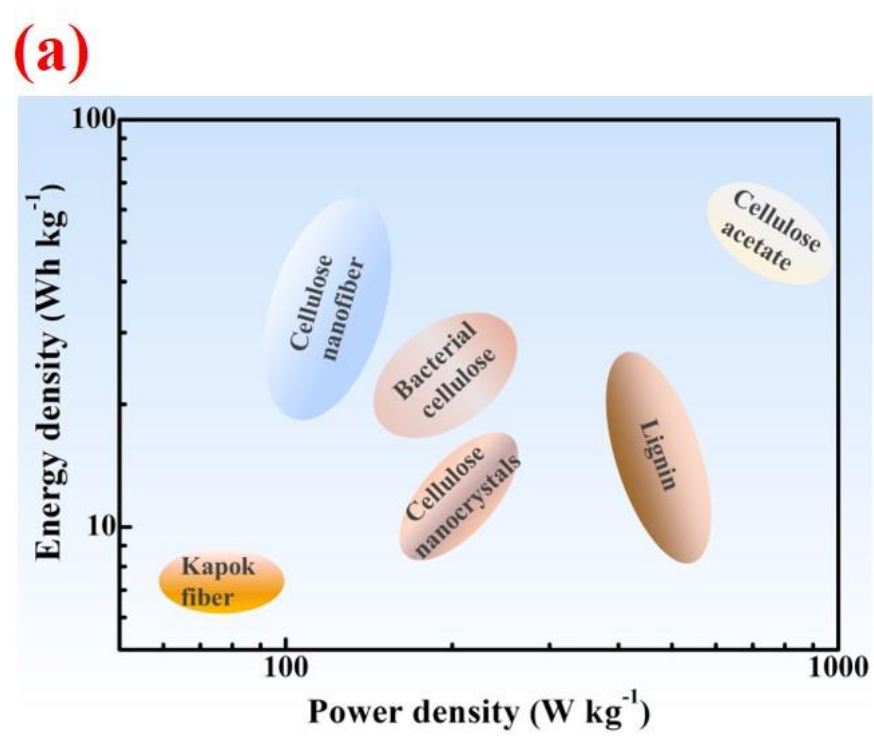

dispersibility and biocompatibility of CNT, and the CNT can also increase the conductivity and thermal stability. ${ }^{[48-50]} \mathrm{Up}$ to now, there are few reviews on the application of flexible cellulose/CNT composite flexible electrode materials in supercapacitors, despite some reviews have been published on nano-cellulose, ${ }^{[51]}$ cellulose nanocrystals, ${ }^{[52]}$ nano-cellulosebased conductive membranes, ${ }^{[53]}$ cellulose-based flexible functional materials, ${ }^{[54,55]}$ and cellulose-based electrochemical energy storage devices. ${ }^{[56]}$ Although the cellulose/CNT composite flexible electrodes have made considerable progress, there still suffer from interface contact and it is a considerable challenge to obtain flexible supercapacitors with high-quality energy/power density, high-quality volume capacity, and long cycle period. Therefore, this review summarizes the preparation, optimization, and research progress of the cellulose/CNT-based composite flexible electrode materials in supercapacitors (Fig. 2), and provides an in-depth outlook on their development prospects in the future.

\section{Preparation method of cellulose/CNT composite flexible electrode material}

The merits of flexible electrode materials determine the performance of flexible supercapacitors. The electrode materials with large specific surface areas and high porosity are the primary selection for electrodes. The cellulose/CNT composite flexible electrode materials with high conductivity, robust mechanical toughness, large specific surface area, and high porosity are a better choice for flexible supercapacitors. The cellulose/CNT composite flexible electrode materials with different properties and morphologies were obtained by different preparation methods. The methods mainly include electrospinning, coating, vacuum filtration, in-situ polymerization, freeze-drying, hot-pressing method, etc. ${ }^{[57-59]}$

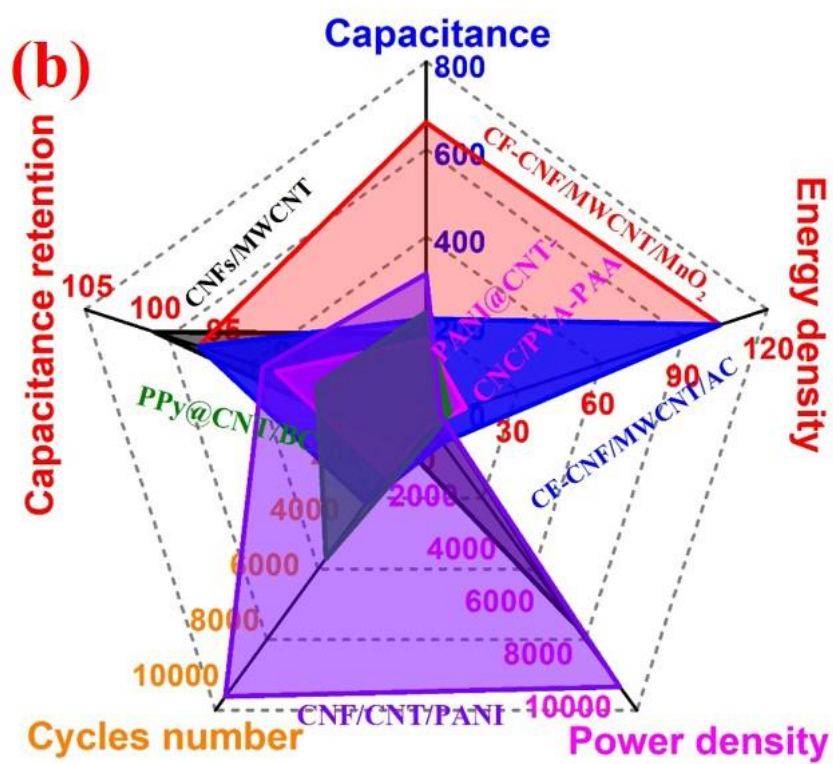

Fig. 1 (a) Ragone diagrams of energy density and power density of cellulose composite electrode materials from different sources in supercapacitors, (b) electrochemical performance of different cellulose/CNT-based composite flexible electrodes in supercapacitors. 


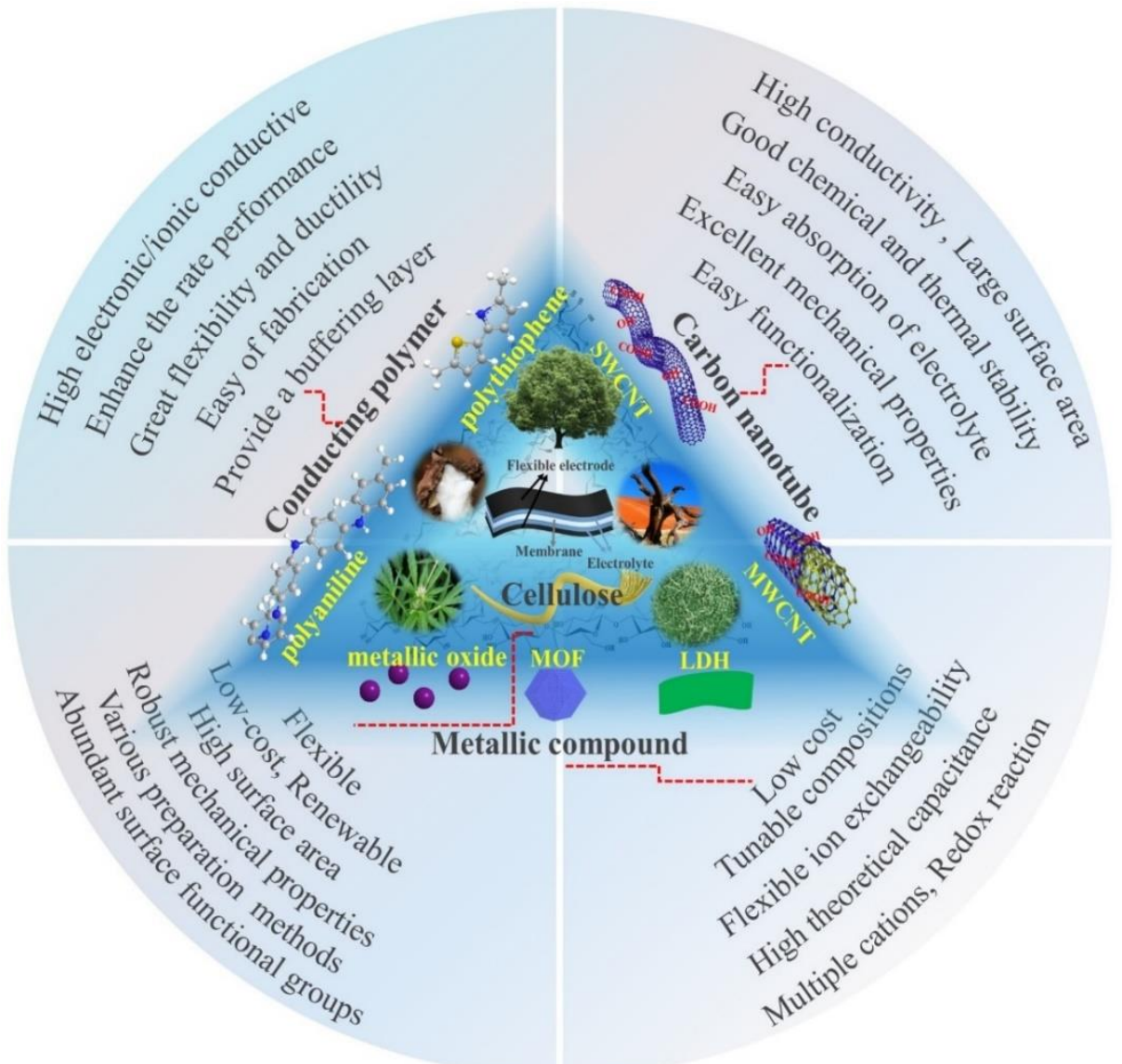

Fig. 2 Schematic illustration of strategies of cellulose/CNT composite flexible electrode materials, and summary of the property advantages of cellulose, carbon nanotube, conducting polymer, and metallic compound for energy storage applications.

\subsection{Electrospinning method}

The electrospinning method is a uniform mixture of cellulose and CNT solution, then through the spinner in a strong electric field for atomization, solidification, and spray spinning into a fine filament with a uniform diameter and controlled structure of cellulose/CNT composite fibers. ${ }^{[00-62]}$ The cellulose/CNT composite nanofibers with flexible and slender structures are beneficial to accelerate the transmission of electrons and improve electrical conductivity, thus enhancing the electrochemical performance of cellulose/CNT composite materials. Cho et al. ${ }^{[63]}$ synthesized tunicate cellulose nanofibril (TCNF)/CNT fibers with good mechanical properties by an electrospinning method (Fig. 3a). The addition of CNT significantly improves the mechanical properties of TCNF. The tensile strength of TCNF/CNT was $240 \mathrm{MPa}$, which is 1.92 times that of TCNF, and the weightbearing capacity of a single fiber from $10 \mathrm{~g}$ (TCNF) to $50 \mathrm{~g}$ (TCNF/CNT). In Fig. 3b, SEM image observation shows that $\mathrm{TCNF} / \mathrm{CNT}$ not only has strong mechanical properties but also has a porous structure that facilitates the transmission of the ions.

Additionally, Deng et al. ${ }^{[64]}$ fabricated multi-walled carbon nanotube (MWCNT)/CNF composite nanofibers with super capacitance via an electrospinning method. The scanning electron microscope (SEM) and transmission electron microscopy (TEM) images of MWNT/CNF showed that some nanotubes protruded on the surface of the nanofiber. The protruding of the nanotubes made the fiber surface rough, thus effectively increasing its surface area. The MWCNT incorporation can reduce the activation energy of MWCNT/CNF composite nanofibers (from 230 to $180 \mathrm{~kJ}$ $\mathrm{mol}^{-1}$ ), and increase the conductivity. The specific capacitance of MWCNT/CNF is 1.38 times that of without MWCNT.

Furthermore, Liu et $a l^{[65]}$ used ionic liquid as a green solvent and dispersant. The highly conductive MWCNTsgraphene sheets (rGOs)-cellulose fibers were prepared via an environmentally friendly wet spinning method. The effects of MWCNTs, rGOs, and cellulose in different ratios on the conductivity, specific surface area, and capacitance of the MWCNTs-rGOs-cellulose fibers were also studied. As shown in Fig. 3c, MWCNTs were dispersed effectively without serious aggregation by cellulose, which was due to the electrostatic repulsion from the interface interaction between oxygen groups on MWCNTs and cellulose. The introduction of rGOs makes the dispersibility of the MWCNTs better, this can be attributed to the $\pi$ stacking effect between MWCNTs and rGOs (Fig. 3d). As shown in Fig. 3e, when the rGOs mass ratio was from 1 increased to 3 , the conductivity of the MWCNTs-rGOs-cellulose fibers can reach $1195 \mathrm{~S} \mathrm{~m}^{-1}$, and the capacitance was increased by 2.27 times. However, the mechanical and electrochemical properties of the prepared cellulose/CNT composite fibers via the electrospinning 

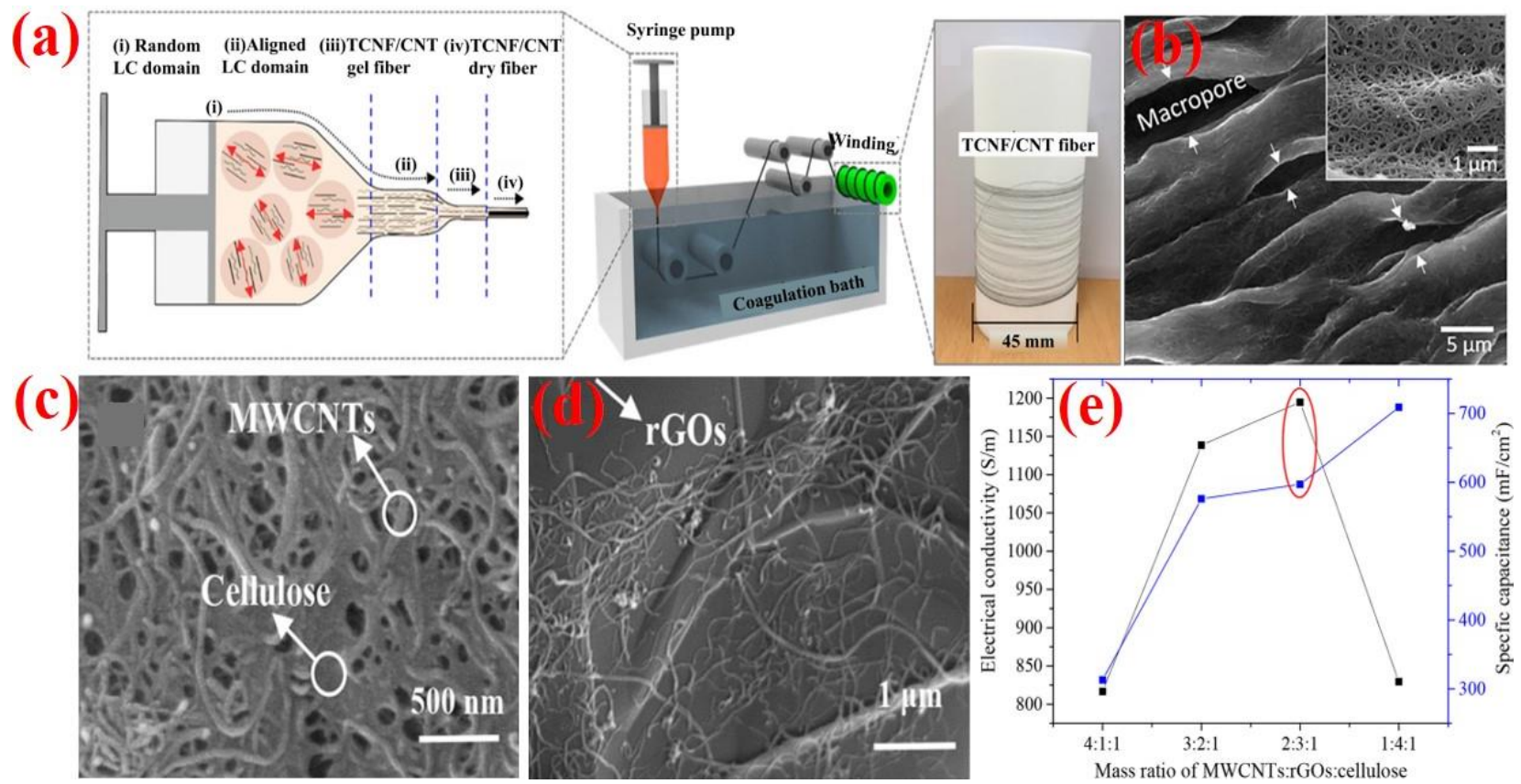

Fig. 3 (a) Schematics of the continuous production of the TCNF/CNT fiber, (b) SEM images of the TCNF/CNT. Reproduced with the permission from [63] Copyright 2019 American Chemical Society; (c) SEM images of 5:0:1 MWCNTs-rGOs-cellulose fiber, (d) SEM images of 2:3:1 MWCNTs-rGOs-cellulose fiber, (e) Effect of MWCNTs and rGOs on the electrical conductivity and specific capacitance. Reproduced with permission from [65] Copyright 2019, American Chemical Society.

methods are affected by many factors such as the physicochemical properties of the solution, electrostatic spinning voltage, speed, the distance between the probe and the receptor surface, humidity, and temperature. Therefore, to design high-performance materials, it is requisite to precisely adjust the above factors.

\subsection{Coating method}

The coating method is in-situ polymerization of cellulose, CNT, conductive polymer, and metal oxide solution. The viscosity of the uniform mixture solution is then reduced via heating, degassing, pressurizing, or stirring. Finally, the lowviscosity mixture solution is then coated/deposited on a silicon wafer or other substrate with a spin coater to obtain a cellulose/CNT composite electrode material. Zhao et al. ${ }^{[66]}$ prepared a highly flexible MWCNTs/cellulose/PEDOT:PSS (MCPP) film by a coating method (Fig. 4a). The incorporation of MWCNTs promotes overcoming the problems of insufficient interface and poor bonding that usually exist between the polymer matrix and MWCNTs. When MWCNT was incorporated into MCPP, the conductivity increased from 26.8 to $275 \mathrm{~S} \mathrm{~m}^{-1}$. In addition, the conductivity remained without destruction even under various bending deformation conditions. The maximum stress and Young's modulus of MCPP film were $95.8 \mathrm{MPa}$ and $3.15 \mathrm{GPa}$, respectively, which were significantly higher than those of cellulose/PEDOT composites.

In Figs. $4 \mathrm{~b}$ and $4 \mathrm{c}$, it can be observed that MWCNTs were uniformly dispersed throughout the cellulose matrix forming a 3D complex network structure. The pore size distribution diagram shows that MCPP has a rich mesoporous structure
(Fig. 4d). The mesoporous structure is conducive to better contact between MCPP and electrolyte, providing abundant ion transmission and diffusion channels, thereby improving the electrochemical performance. The coating method is a way to obtain cellulose/CNT composites with high electrical conductivity and good mechanical properties without changing the crystalline structure of cellulose. ${ }^{[67,68]}$ Compared with the electrospinning method, the coating method has the advantages of simple operation and low economic cost. However, it still has its disadvantage, that is, the interface contact is not close enough, and slightly lower mechanical properties of the composite material are observed.

\subsection{Vacuum filtration method}

The vacuum filtration method has the advantages of simple and flexible operation, small footprint, low economic cost, and high efficiency, and it has attracted great attention in the preparation of membrane/paper electrodes. ${ }^{[69-71]}$ Fig. 5a shows the schematic of CNF/CNT film by the vacuum filtration method. Chen et al. ${ }^{[72]}$ mixed CNF and MWCNT solution with different mass ratios, and obtained highly conductive $\mathrm{CNF} / \mathrm{MWCNT}$ gel film through vacuum filtration, dehydration, $\mathrm{NaOH}$ impregnation, and freeze-drying. The conductivity of CNF/MWCNT gel film was $17.04 \mathrm{~S} \mathrm{~cm}^{-1}$, which was lower than that reported by electrostatic spinning and coating method. Subsequently, the effect of MWCNT on membrane shrinkage was studied, and the test results showed that the shrinkage increased with the decrease in MWCNT content. When the content of MWCNT was excessive $(\geq 50$ $\mathrm{wt} \%$ ), the protrusions were present on the surface of the CNF/MWCNT film. The protrusion increases the contact 


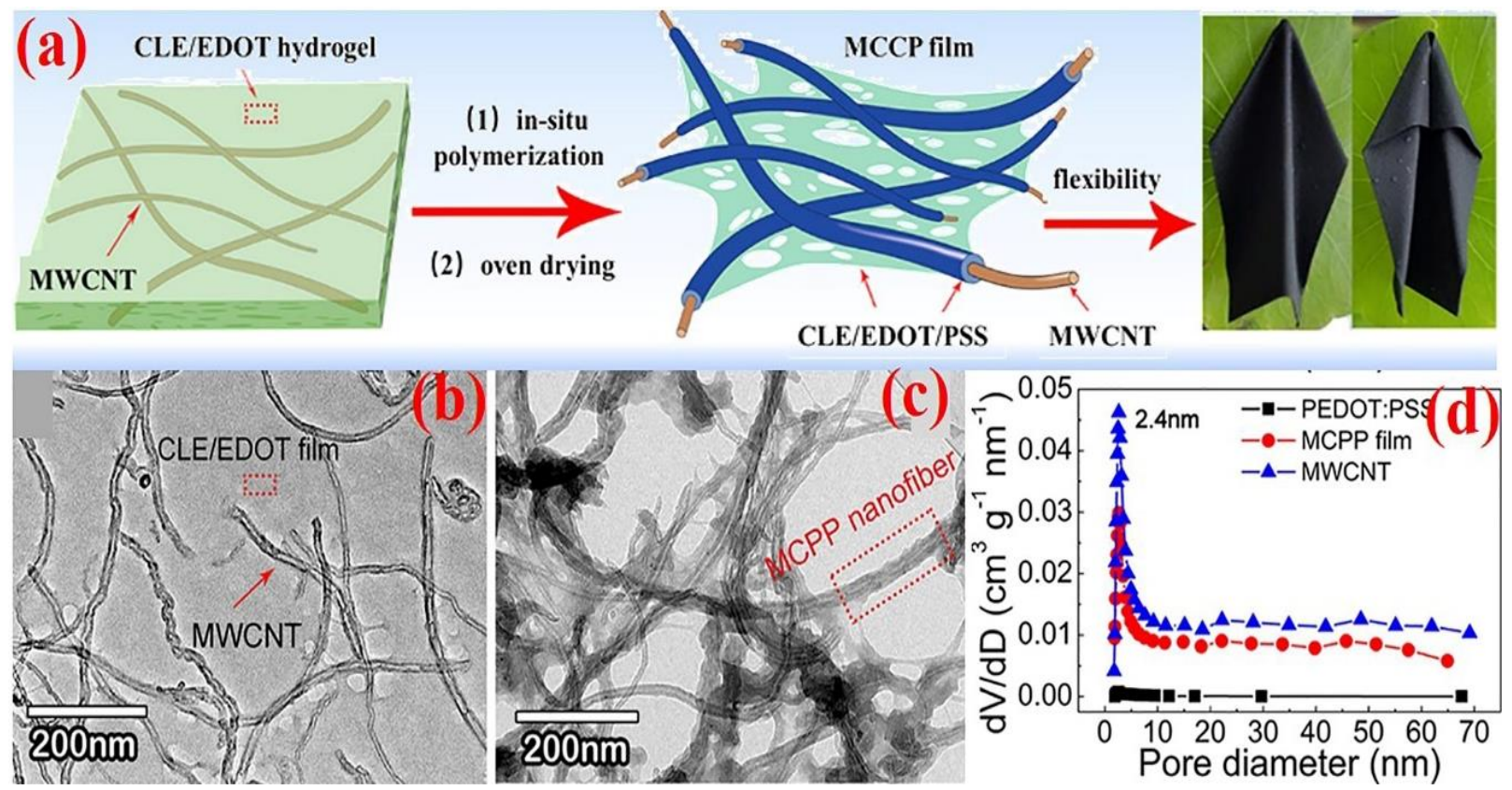

Fig. 4 (a) Schematic illustration of the MCPP films, (b, c) TEM images showing the MWCNTs in the cellulose/EDOT system, and (d) pore size distribution of the MCPP film. Reproduced with permission from [66] Copyright 2017 American Chemical Society.

distance of the composite film material interface, obstructs the electron transmission channel, and reduces its electrochemical performance.

Unlike previous reports in the literature, Qu et al. ${ }^{[73]}$ used nano-cellulose as the substrate and binder of flexible film, and innovatively designed a binder-free nano-cellulose/carbon nanotubes composite flexible film electrode (NPCC) with high conductivity by vacuum filtration method. In Figs. 5b-e, the NPCC flexible film electrode can still return to its original shape after bending and twisting, indicating that NPCC has excellent flexibility, and has potential applications in the field of flexible devices. In summary, the preparation of flexible electrodes by vacuum filtration method has a good application prospect in supercapacitors, but there are problems, for example, uneven material distribution, easy agglomeration of conductive materials, and limited interface leading to material bonding, resulting in low cycle stability performance of the flexible electrode material. Thus, the prepared cellulose/CNT composites have a tighter interfacial contact by adding surfactants or optimizing the modification of the surface of cellulose and CNT materials. This can eliminate the interfacial resistance and enhance the electrochemical performance of the cellulose/CNT composite flexible electrodes.

\subsection{Other preparation methods}

In addition to the above methods, freeze-drying, hot pressing, extrusion molding, and layer-by-layer self-assembly method were also used to prepare cellulose/CNT composite flexible electrode materials.[ 74 ] Oriented porous CNT/cellulose composites were prepared by a freeze-drying method. ${ }^{[75]}$ The

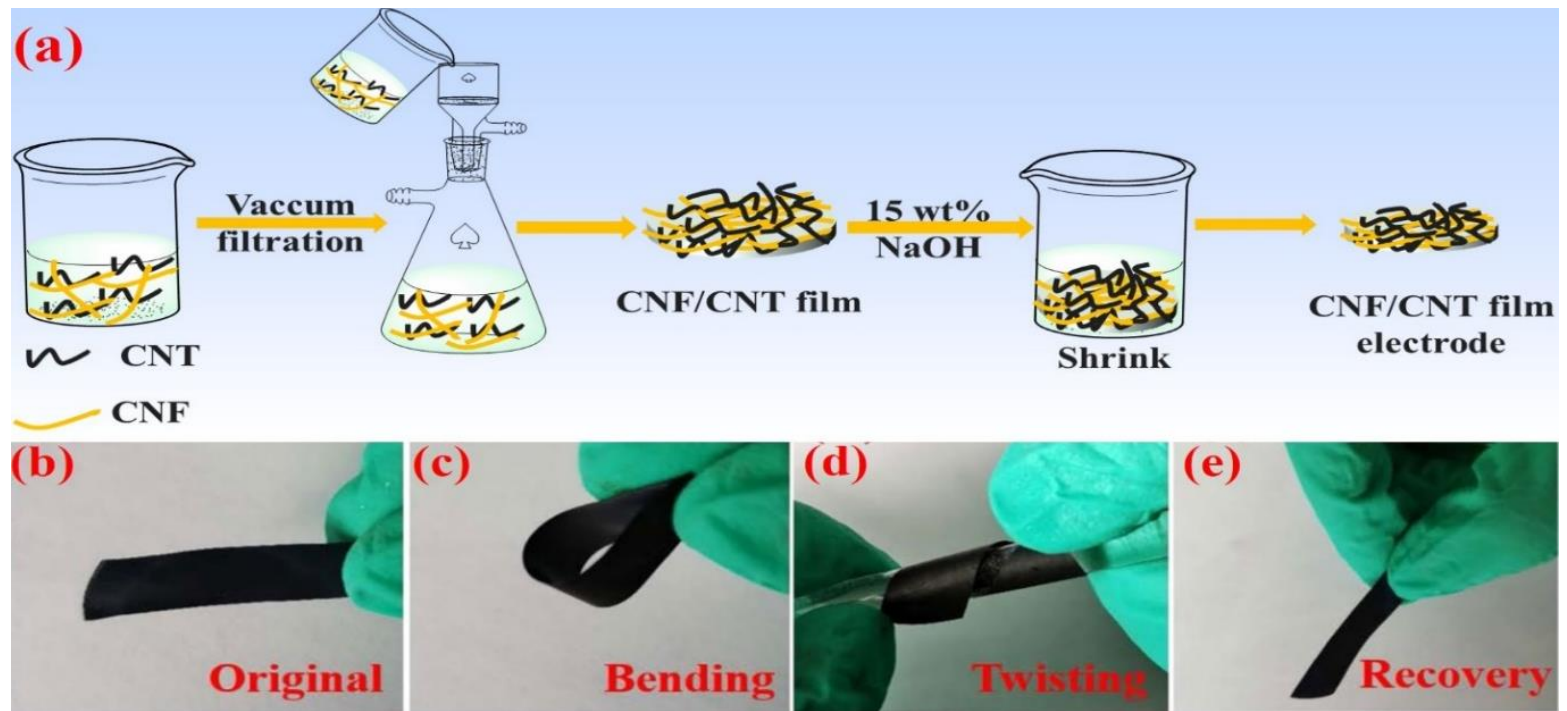

Fig. 5 (a) Schematic of preparation of CNF/CNT film, (b-e) flexibility test of NPCC. Reproduced with permission from [73] Copyright 2021 Springer Nature Switzerland AG. 
method includes the processes of dissolution, one-way freezing, freeze-drying and etching, and CNT-matrix/cellulose porous composite and CNT-interface/cellulose porous composite were synthesized through two paths, respectively (Fig. 6a). With the increase of CNT content, the conductivity of the two composite materials continues to increase. When the CNT loading increased from 0 to $0.17 \mathrm{vol} \%$, the conductivity of the composite material increased by nearly $10^{13}$ orders of magnitude. In Fig. 6b, the TEM image of CNTinterface/cellulose porous composite, CNTs were packed tightly in a limited space-the surface of the cellulose cell wall. The interconnected CNT network makes the composite material highly conductive.
Xia et al. ${ }^{[45]}$ prepared carbon fiber-reinforced cellulose nanofiber/CNT-hybrid aerogels (CF-CNF/MWCNT-HAs) by a freeze-drying method. Subsequently, $\mathrm{KMnO}_{4}$ was added into the CF-CNF/MWCNT-HAs, and the CNF/MWCNT/MnO ${ }_{2-}$ HAs were obtained via freeze-drying and annealed synthesis. The activated carbon (AC) was added into the CNF/MWCNT solution in the same way, and the CF-CNF/MWCNT/AC-Has were obtained via the processes of extrusion and annealing. In composite aerogels materials, the aerogel embedded with cellulose nanofiber (CF) has a honeycomb-like threedimensional porous structure, which can avoid structural damage and collapse caused by the $\mathrm{KMnO}_{4}$ solution. Benefiting from the structural support and strength of the

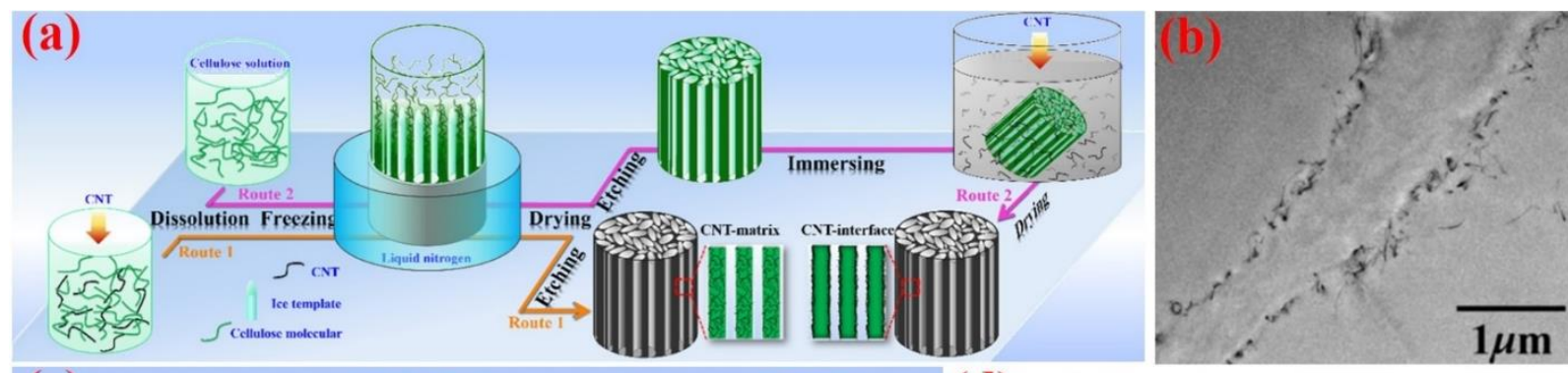

(c)

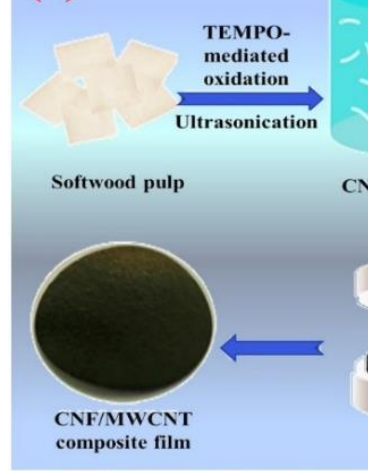

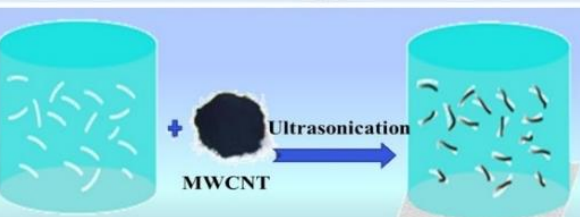

CNF dispersion
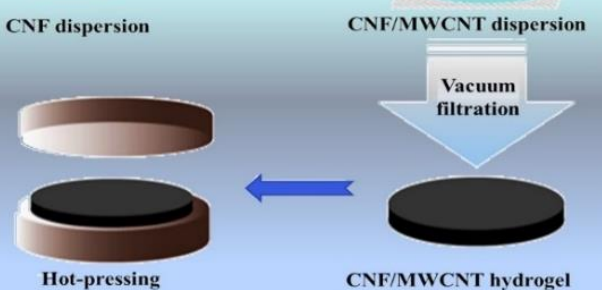

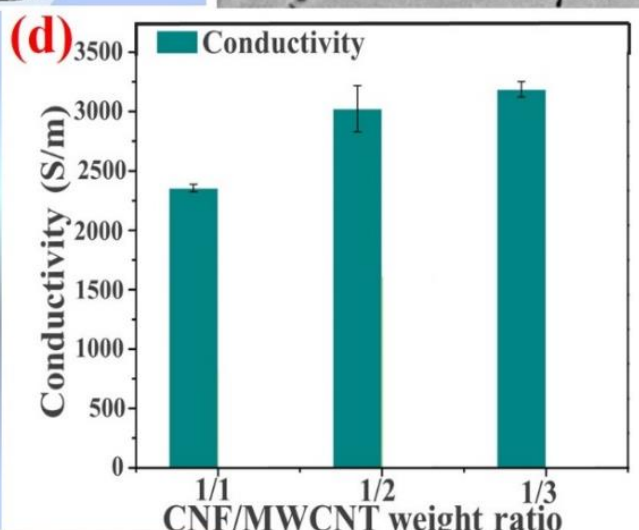

(e)
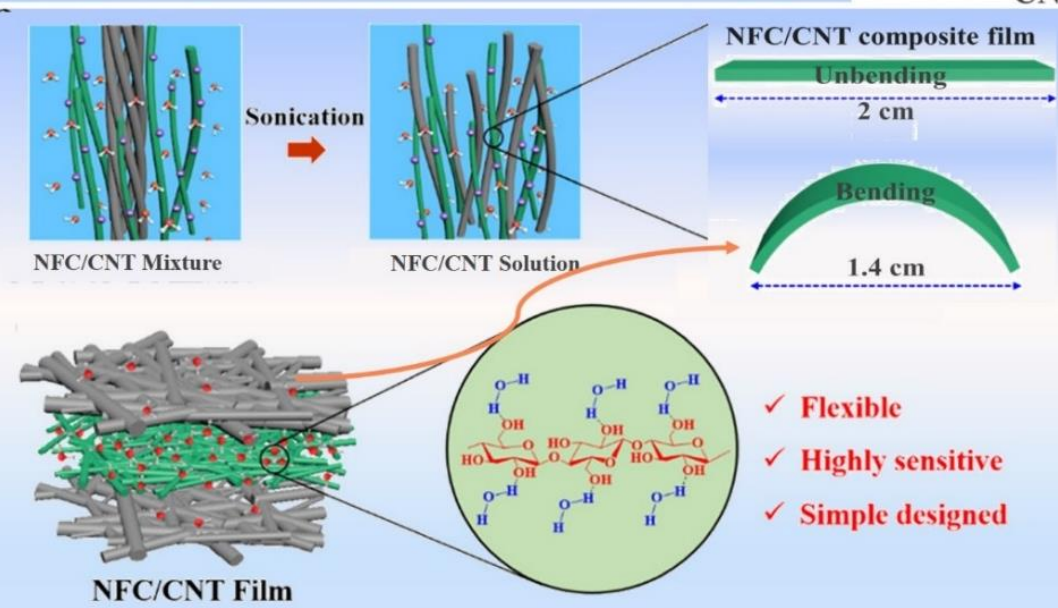

Mwc

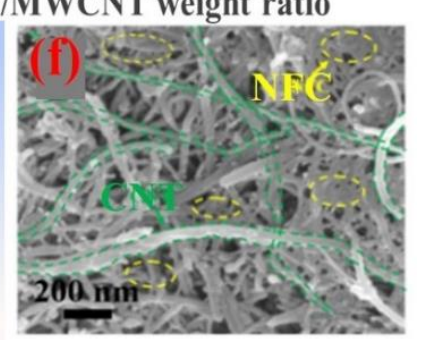

$=\mathrm{CNT}-\mathrm{NFC}$ - Water molecule $\odot \mathrm{COO}$ group $\odot$ Carbon atom Hydrogen atom

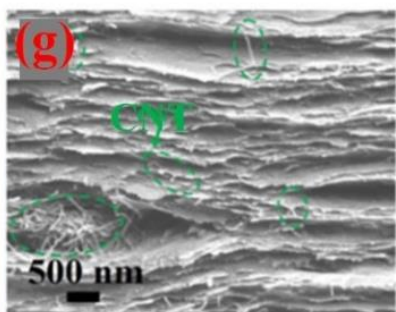

Fig. 6 (a) Schematic illustration of the preparation process of CNT/cellulose porous composite, (b) TEM images of CNTmatrix/cellulose porous composite. Reproduced with permission from [75] Copyright 2018 American Chemical Society; (c) schematic of fabrication of CNF/MWCNT composite film, (d) conductivity of CNF/MWCNT composite films with different weight ratios. Reproduced with permission from [68] Copyright 2018 American Chemical Society; (e) schematic illustrating the dispersion mechanism of CNTs by NFC and the hierarchical structure of the obtained NFC/CNT film. (f) SEM images show the NFC/CNT network structure and (g) the layer-by-layer structure of the CNT film. Reproduced with permission from [59] Copyright 2019 American Chemical Society. 
uniformly arranged $\mathrm{CF}$, the aerogel does not collapse in structure. The electrochemical tests found that the capacitance of the CF-CNF/MWCNT/MnO 2 and CF-CNF/MWCNT/AC electrodes was 1745 and $1273 \mathrm{mF} \mathrm{cm}^{-2}$ at the current density of $1 \mathrm{~mA} \mathrm{~cm} \mathrm{~cm}^{-2}$, respectively. The cellulose/CNT composite electrodes by a freeze-drying method not only maintain the original chemical composition and morphology but also can produce porous structures that are conducive to the transport and adsorption of electrolyte ions. ${ }^{[76]}$

Additionally, the hot-pressing method due to its simple operation, high efficiency and can be mass-produced also was used to prepare cellulose/CNT composite electrode materials. As shown in Fig. 6c, the MWCNT and CNF suspension by ultrasound and vacuum filtration obtained a stable MWCNT/CNF hydrogel, and then via hot pressing to obtain CNF/MWCNT composite membrane electrodes with high conductivity. ${ }^{[69]}$ The conductivity of the CNF/MWCNT films with different mass ratios was measured via the four-point probe method (Fig. 6d). When the mass ratio of $\mathrm{CNF}$ and MWCNT was $1: 1$, the conductivity of the films reached 2356 $\mathrm{S} \mathrm{m}^{-1}$. This result indicated that the conductivity of cellulose increases with the increase of MWCNT content, which also proves that the addition of CNT can solve the conductivity of cellulose. This membrane electrode with abundant - $\mathrm{OH}$, $\mathrm{COOH}$, hydrophilic functional groups, and a large specific surface area facilitate electron transport and transfer, increasing electrical capacity, and enhancing its electrochemical performance in electronic and sensor devices.

In Fig. 6e, Zhu et al. ${ }^{[59]}$ prepared the nano-fibrillated cellulose (NFC)/CNT flexible composite film with network and layer-by-layer structure by the layer-by-layer selfassembly method. The NFC/CNT film is highly flexible and can be bent up to $1.4 \mathrm{~cm}$ for a $2 \mathrm{~cm}$ film. As shown in Figs. 6f, $\mathrm{g}$, the SEM images of $\mathrm{NCF} / \mathrm{CNT}$ flexible film indicated that $\mathrm{NCF} / \mathrm{CNT}$ film not only has a good network structure but also has a layer-by-layer structure. Additionally, the Fouriertransform infrared (FTIR) test showed that NCF and CNT were bonded by hydrogen bonds, and this non-covalent interaction made the interfacial contact of the composite film closer. It is noteworthy that the NCF/CNT flexible film prepared by the layer-by-layer method can be controlled to a thickness of $24 \mu \mathrm{m}$.

Overall, all of the above preparation methods have their own unique advantages and disadvantages. The electrical conductivity will be improved after the composite of cellulose and CNT. However, excessive CNT ( $\geq 30 \mathrm{wt} \%$ ) will reduce the mechanical properties of cellulose/CNT composites, and make them easy to fracture and strain failure. Therefore, controlling the amount of both is also an important condition for the preparation of cellulose/CNT composites with excellent properties.

\section{Application of cellulose/carbon nanotube composite flexible electrode for supercapacitors}

Cellulose/CNT electrode materials have the problems of small capacitance, low mass/volume energy density, and poor cycle stability, which hinder their large-scale applications. Consequently, many studies by constructing richer pore structures, increasing the specific surface area, and combining with pseudo-capacitance materials (such as conductive polymers, metal oxides, and metal hydroxides) effectively improve the electrochemical performance of the supercapacitor. Wherein, pseudo-capacitance materials can be distributed on the surface and inside of the electrode, and the pseudo-capacitance can be generated via the reversible chemical adsorption and desorption or redox reaction. More interestingly, the synergy between the pseudo-capacitance and the electric double-layer capacitance of the cellulose/CNT itself was used to improve the electric capacity and energy density of the cellulose/CNT composite. Additionally, the incorporation of pseudo-capacitance materials can increase the adsorption capacity of the electrolyte, and reduce the interface resistance between the electrode and the electrolyte to enhance the electrochemical stability, rate, and cycle stability of the electrode. ${ }^{[38,74,77,78]}$ Next, this section mainly focuses on the applications of cellulose/CNT composite flexible electrodes of different forms (e.g. gel flexible electrodes, composite membrane flexible electrodes, and paper-based flexible electrodes, etc.) for supercapacitors.

\subsection{Gel flexible electrode}

The gel is a kind of colloid with certain elasticity, strength, inherent characteristics, and three-dimensional network structure. Cellulose interacts with the cross-linking agent to form a cellulose hydrogel with spatial networks. Then the aerogels can be obtained from hydrogel via freezing or supercritical $\mathrm{CO}_{2}$ drying. The high porosity and orderly space structure of the gel are beneficial to the transport of electrons and the storage of electrolytes. Han et al. ${ }^{[79]}$ used CNF as a template and CNT dispersant to prepare a CNT$\mathrm{CNF} /$ polyvinyl alcohol borax (PVAB) composite hydrogel with high electrical conductivity, viscoelasticity, and excellent supercapacitor. The specific capacitance of the all-solid mechanical toughness, and moldability. The compressive stress of CNT-CNF/PVAB composite hydrogel was $\sim 93 \mathrm{kPa}$, and the storage modulus was $\sim 7.12 \mathrm{kPa}$, which were 2.7 times and 1.9 times of $\mathrm{CNF} / \mathrm{PVAB}$ hydrogel, respectively. In addition, the CNT-CNF/PVAB composite hydrogel has $20 \mathrm{~s}$ self-healing ability and high electrical conductivity. The CNT$\mathrm{CNF} / \mathrm{PVAB}$ hydrogel was used as an electrode material and a self-repairing solid supercapacitor was assembled. The supercapacitor has a higher capacitance of $117.1 \mathrm{~F} \mathrm{~g}^{-1}$, and a good capacitance retention rate of $96.4 \%$ after 1000 cycles. After ten destruction-self-repair cycles, there is still an ideal capacitance retention rate of $98.2 \%$. Additionally, for the selfrepairing solid supercapacitor in the state of bending, folding, and twisting, the capacitance retention rate can reach about 95\% after 1000 cycles.

Gel-prepared electrodes can eliminate the problems of increased interfacial impedance, decreased mass transfer 
efficiency, and electrochemical performance caused by the binder. For example, Xia et al..$^{[45]}$ used carbon fiber-reinforced cellulose nanofiber/MWCNT mixed aerogels (CFCNF/MWCNT-HAs) as templates to prepare a binder-free, highly flexible, good mechanical, and three-dimensional porous structure of aerogels electrodes (CF$\mathrm{CNF} / \mathrm{MWCNT} / \mathrm{MnO}_{2}$ and $\left.\mathrm{CF}-\mathrm{CNF} / \mathrm{MWCNT} / \mathrm{AC}\right)$. The CF$\mathrm{CNF} / \mathrm{MWCNT} / \mathrm{MnO}_{2}$ and $\mathrm{CF}-\mathrm{CNF} / \mathrm{MWCNT} / \mathrm{AC}$ were used as the positive and negative electrodes to construct $\mathrm{CF}$ $\mathrm{CNF} / \mathrm{MWCNT} / \mathrm{MnO}_{2} / / \mathrm{CF}-\mathrm{CNF} / \mathrm{MWCNT} / \mathrm{AC}$ flexible asymmetric supercapacitors (ASC). The volume capacitance and energy density of flexible ASC were $19.4 \mathrm{~F} \mathrm{~cm}^{-3}$ and 8.93 $\mathrm{mWh} \mathrm{cm}^{-3}$, respectively. In addition, the flexible ASC can retain more than $96.7 \%$ capacity after 3000 cycles.

Similarly, Gao et al. ${ }^{[44]}$ were fabricated CNFs/MWCNTs hydrogel with good wettability and high mesopore utilization rate by the supercritical $\mathrm{CO}_{2}$ drying method. The CNFs/MWCNTs hydrogel as an electrode and current collector with a sulfuric acid-polyvinyl alcohol gel $\left(\mathrm{H}_{2} \mathrm{SO}_{4-}\right.$ PVA) electrolyte to assemble an all-solid flexible supercapacitor was $178 \mathrm{~F} \mathrm{~g} \mathrm{~g}^{-1}$ at the scan rate of $5 \mathrm{mV} \mathrm{s}^{-1}$, and the energy density and power density were $5.06 \mathrm{Wh} \mathrm{kg}^{-1}$ and $7.67 \mathrm{~kW} \mathrm{~kg}^{-1}$, respectively. CNF/MWCNTs aerogel electrode has good electrochemical performance, high faraday efficiency (96.8\%), and excellent circulation stability, but also is green and environmentally friendly.

To further prepare flexible supercapacitors with high area capacitance, area power density, and energy density. Zheng et al. ${ }^{[33]}$ used water-soluble graphene oxide nanosheets (GONSs) as surfactants to disperse CNTs, and prepared $\mathrm{CNF} / \mathrm{GONS} / \mathrm{CNT}$ gel electrodes by freeze-drying methods (Fig. 7a). As illustrated in Figs. 7b, c, the all-solid-state supercapacitors were assembled using the $\mathrm{CNF} / \mathrm{GONS} / \mathrm{CNT}$ gel electrodes as the anode and the cathode, and $\mathrm{H}_{2} \mathrm{SO}_{4} / \mathrm{PVA}$ gel as the electrolyte. It is particularly worth noting that three all-solid-state supercapacitors in series can light up LED bulbs with an electric voltage of $3 \mathrm{~V}$ (Fig. 7d). The electrochemical performance of all-solid-state supercapacitor under the bending state is an important index to evaluate their practical applications. Fig. 7e displays the CV curves of the all-solidstate supercapacitor under different bending angles. The results show that the bending angle has no significant effect on the capacitance performance, indicating that an all-solid-state supercapacitor has excellent fleshiness and electrochemical performance. In addition, the self-discharge of the all-solidstate supercapacitor was explored. Fig. $7 \mathrm{f}$ shows the selfdischarge curve of the supercapacitor after charging it at $1.0 \mathrm{~V}$ for $10 \mathrm{~min}$. Even after $24 \mathrm{~h}$, the initial charging potential remained close to $59 \%$. The results demonstrated that the device has practicability.

In summary, based on the advantages of cellulose and CNT, the cellulose/CNT compound gel has good electrochemical performance when used as a flexible electrode material. Whereas, there are still some problems, such as limited charge adsorption, storage space, low energy density, and difficulty in achieving an order-of-magnitude increase in capacitance. Reasonably optimizing the pore structure and increasing the specific surface area of cellulose/CNT compound gel is beneficial to better matching the electrolyte ions and thus reaching an improved electrochemical performance.

\subsection{Composite membrane flexible electrode}

Cellulose membranes have the advantages of large specific surface areas, high porosity, strong mechanical properties, and good electrolyte wettability. Therefore, they were used as flexible electrode substrates by in-situ polymerization compounded with metal or a conductive polymer. ${ }^{[80]}$ The CNT has abundant carboxyl and hydroxyl functional groups, and

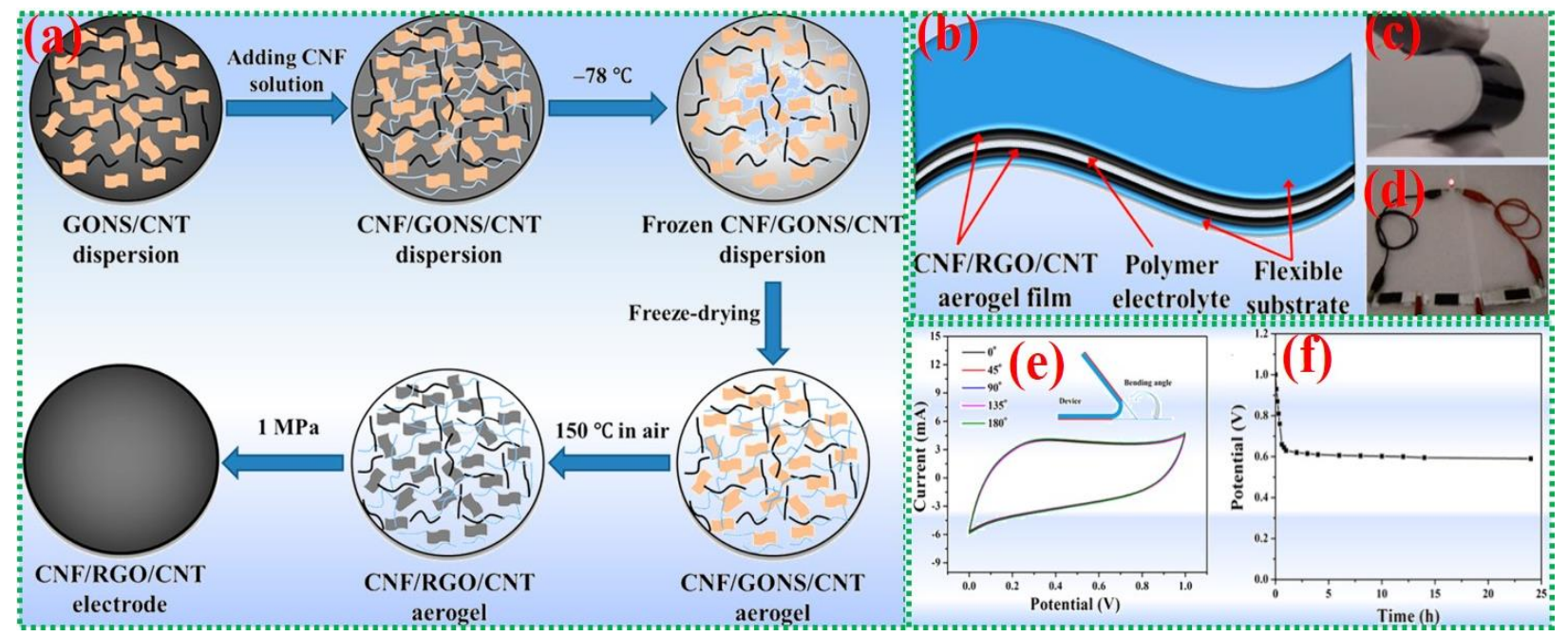

Fig. 7 (a) Schematic of the fabrication process of a CNF/RGO/CNT electrode, (b) Schematic diagram of the all-solid-state supercapacitor where the polymer-gel electrolyte serves as the electrolyte and the separator. Inset shows, (c) Optical picture of the flexibility of the device, (d) Three highly flexible devices used in series to light a red LED, and (e) the Dependence of specific capacitance for a supercapacitor at different bending angles. The inset shows the charge-discharge curves of a supercapacitor at different bending angles with a current density of $1 \mathrm{~A} \mathrm{~g}^{-1}$. (f) Self-discharge curves of a supercapacitor after being charged at $1.0 \mathrm{~V}$ for 10 min. Reproduced with permission from ${ }^{[33]}$ Copyright 2015 American Chemical Society. 
thus the CNT can be combined with the cellulose membrane by electrostatic and hydrogen bonding to obtain the cellulose/CNT conductive composite membrane material with a good interface effect. The incorporation of CNT promotes stress transfer in the interface area, reduces the hydrogen bond density within and between the cellulose molecules, and enhances the toughness and plasticity of composite materials. ${ }^{[81]}$ To increase the force between the electrode and the electrolyte, reducing the diffusion resistance of electrolyte ions, and improve its electrochemical performance, many studies have been devoted to the addition of pseudocapacitance materials.

To further improve the capacitance and cycle stability of the cellulose/CNT-based membrane electrode, Jyothibasu et al. ${ }^{[82]}$ prepared polypyrrole (PPy)/f-CNT/cellulose composite membrane electrode with high conductivity, good wettability, and rich pore structure via loading PPy onto a functionalized CNT ( $f$-CNT) and regenerated cellulose substrates by in-situ polymerization methods. The membrane electrode with a rich pore structure and large specific surface area was beneficial to the storage/release of charge carriers and the diffusion of electrolyte ions. The capacitance of the PPy/f-CNT/cellulose composite membrane electrode was $2147 \mathrm{mF} \mathrm{cm}^{-2}$ at the current density of $1 \mathrm{~mA} \mathrm{~cm}^{-2}$. Additionally, it can cycle up to 5000 cycles at the current density of $20 \mathrm{~mA} \mathrm{~cm}^{-2}$, which indicates its excellent cycling stability.

High thermal stability, good mechanical tenacity, strong water resistance, and excellent electrochemical performance are the development goals of cellulose-based composite membrane electrodes. For example, Han et al. ${ }^{[31]}$ doped CNT and CNC into the mixed solution of PVA and polyacrylic acid (PAA). The CNT-CNC/PVA-PAA nanofiber membranes were obtained by the following directional electrospinning, heat treatment, and cross-linking. The CNT-CNC/PVA-PAA nanofiber membrane was also used as a core material, then via thermally induced esterification and in-situ polymerization of the PANI shell layer to obtain PANI@CNT-CNC/PVA-PAA membrane electrode with high strength, good thermal stability, strong water resistance, and mechanical properties. The PANI@CNT-CNC/PVA-PAA nanofiber membrane has high porosity, large specific surface areas, high tensile strength $(\sim 54.8 \mathrm{MPa})$, good electrical conductivity $\left(\sim 0.44 \mathrm{~S} \mathrm{~m}^{-1}\right)$, and flexibility. As shown in Fig. 8a, the PANI@CNT-CNC/PVAPAA membrane electrode was combined with a gel electrolyte (PVA-g-PAA-KCl) to assemble a flexible supercapacitor. The assembled flexible supercapacitors have high energy density $\left(13.8 \mathrm{Wh} \mathrm{kg}^{-1}\right)$ and good stability (capacitance retention rate is $91 \%$ after 2000 cycles). It is worth noting that the conductivity of the membrane electrode was still relatively stable even under bending and twisting deformation (Fig. 8b). As illustrated in Fig. 8c, for the symmetrical solid supercapacitors assembled by PANI@CNT-CNC/PVA-PAA electrodes under the conditions of the plane, bending, and twisting deformation, after 2000 cycles, the capacitance retention rates were $92 \%, 90 \%$, and $89 \%$, respectively.
The above researches show that the cellulose composite membrane electrodes have potential development prospects in the fields of flexible and foldable supercapacitors and sensors. ${ }^{[83]}$ Compared with $\mathrm{CNF}$ and $\mathrm{CMF}, \mathrm{CNC}$ has relatively low length and diameter, high crystallinity, and rigid particles that are difficult to be disentangled to form a network structure, so the film-forming ability is poor, and there is less research in the field of energy storage. Particularly, CNC can reduce the problem of water in charge storage devices, making it particularly promising for use in charge storage devices.

\subsection{Paper-based flexible electrode}

The highly durable and flexible paper electrodes have attracted wide concern in the field of flexible electrodes for supercapacitors, owing to their low cost, biodegradability, high ion accessibility, and flexibility. Wu et al. ${ }^{[84]}$ embedded $\mathrm{BC}$ in a cellulose fiber matrix to obtain the paper substrate with a dual fiber matrix structure (Fig. 9a). The BC not only facilitated the adsorption, stabilization, and dispersion of CNTs, but also reduced costs, improved the rigidity of the material, and enhanced the accessibility of electrolyte ions. The paper electrode has a conductivity of $5.9 \times 10^{-1} \mathrm{~S} \mathrm{~cm}^{-1}$, which was almost constant even under high bending strain. The electrochemical performance of paper electrodes in different electrolytes was also discussed in this work. As shown in Figs. 9b and c, in the $\mathrm{H}_{2} \mathrm{SO}_{4}$ electrolyte, the paper electrode has a specific capacitance of $77.5 \mathrm{~F} \mathrm{~g} \mathrm{~g}^{-1}$, and the capacitance remains above $98 \%$ after 15,000 cycles. In addition, under the conditions of organic electrolyte, $2 \mathrm{~V}$ potential windows, and $1 \mathrm{~A} \mathrm{~g}^{-1}$ current density, the energy density and power density of the paper electrode reached 22.5 $\mathrm{Wh} \mathrm{kg}^{-1}$ and $873 \mathrm{~kW} \mathrm{~h}^{-1}$, respectively.

Electrode materials with large capacitance, good rate, highquality energy density, and excellent cycle stability are the directions of efforts in the field of flexible supercapacitors. For example, Etman et al. ${ }^{[85]}$ embedded metal oxide $\left(\mathrm{MoO}_{3-\mathrm{x}}\right)$ into CNT and dendritic cellulose matrix by ultrasonic and vacuum filtration and prepared a flexible paper electrode $\left(\mathrm{MoO}_{3-\mathrm{x}^{-}}\right.$ CNTs-CC) with a thickness of $12 \mu \mathrm{m}$ (Figs. 9d, e). The addition of $\mathrm{MoO}_{3-\mathrm{x}}$ increases electrode conductivity and pseudo-capacitance. As displayed in Fig. 9f, the capacitance of the $\mathrm{MoO}_{3-\mathrm{x}}-\mathrm{CNTs}-\mathrm{CC}$ paper, the electrode was $30 \mathrm{C} \mathrm{g}^{-1}$ at a high current density of $78 \mathrm{~A} \mathrm{~g} \mathrm{~g}^{-1}$. After 30,000 cycles, the capacitance retention rate reaches $91 \%$. This result indicates that the $\mathrm{MoO}_{3-\mathrm{x}}-\mathrm{CNTs}-\mathrm{CC}$ flexible paper electrode still has good electrochemical stability at high charge/discharge rates. In the era of rapid development of demand for wearable smart energy storage devices, flexible supercapacitors must have the ability to be repeatedly folded while having excellent electrochemical performance. For example, Ge et al. ${ }^{[36]}$ used the network of macro-porous cellulose fiber as the scaffold, and the SWCNT was wrapped around the cellulose fiber to form a conductive surface layer by an impregnationabsorption-polymerization method. Then the PANI nanobelt was polymerized via the in-situ method to prepare the 

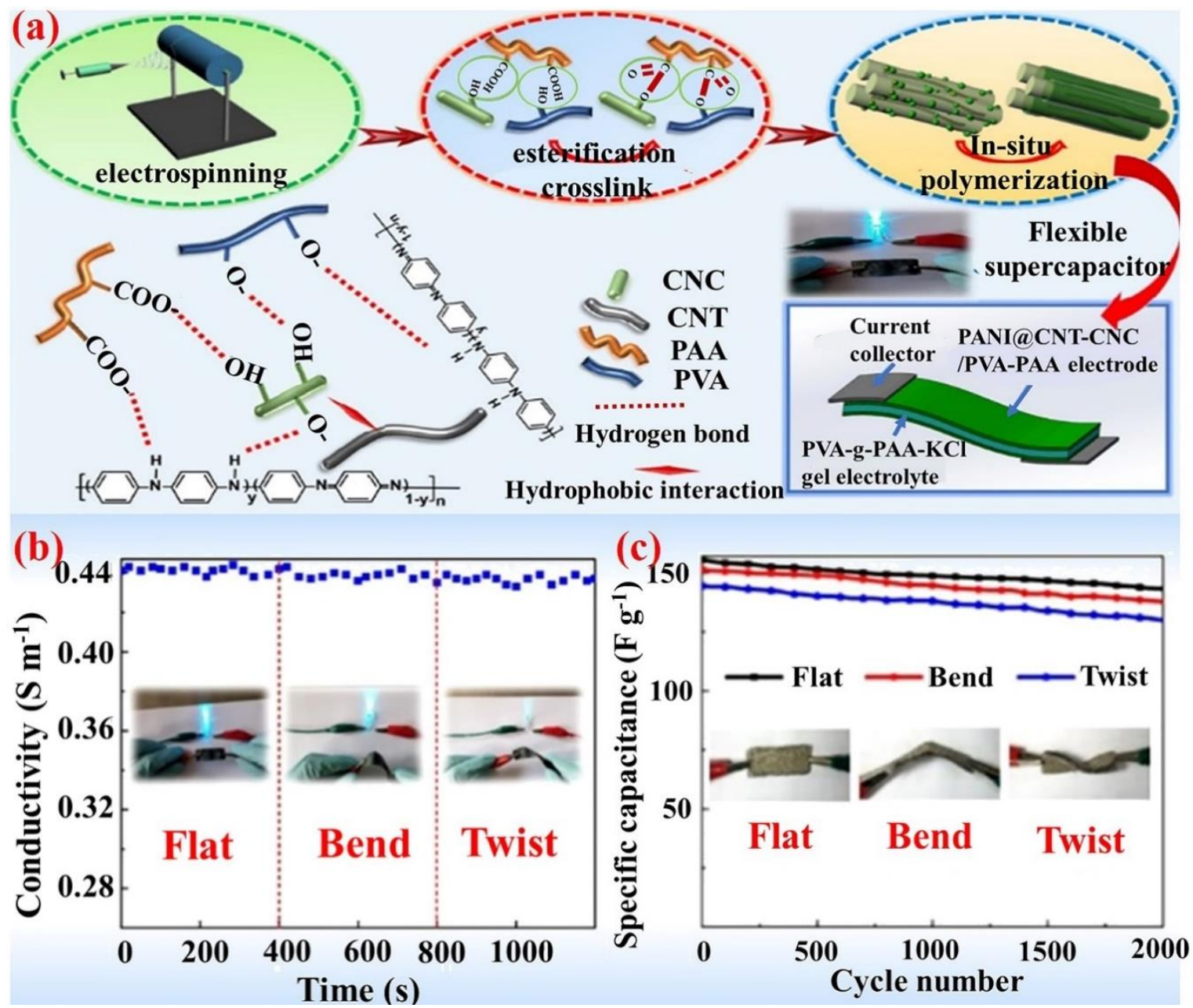

Fig. 8 (a) Schematic illustration of the symmetrical flexible supercapacitor, (b) electroconductivity stability of the PANI@CNTCNC/PVA-PAA electrode under various mechanical deformations, and (c) cycling life tests performed at $1.0 \mathrm{~A} \mathrm{~g}^{-1}$ for the flexible supercapacitor and the inset demonstrating its cyclic stability under flat, bending, and twisting deformations. Reproduced with permission from [31] Copyright 2019 American Chemical Society.

SWCNT/PANI nanoribbon paper electrodes. These paper electrodes were assembled into a foldable flexible supercapacitor. The network structure of SWCNT can shorten the electron transmission path. In addition, the synergistic effect of SWCNT and PANI nanobelt accelerates the charge transfer, resulting in electrode material having good volume capacitance $\left(40.5 \mathrm{~F} \mathrm{~cm}^{-3}\right)$ and area capacitance $\left(0.33 \mathrm{~F} \mathrm{~cm}^{-2}\right)$. The SWCNT/PANI nanobelt paper electrode was folded into a paper crane 1000 times, and there was no mechanical failure or loss of capacitance, indicating high flexibility and compliance. Six all-solid-state supercapacitors were connected in series to light up the LED bulb. Additionally, it is found that the LED bulb can be lit before, during, and after the folding.

Noremberg et al. ${ }^{[86]}$ assembled a supercapacitor based on cellulose, carboxylated CNT, and ionic liquid. The conductive paper electrode had a capacitance of $34.37 \mathrm{~F} \mathrm{~g}^{-1}$ under the high potential window of $0 \sim 2 \mathrm{~V}$. The capacitance retention rate was $97.9 \%$ after 5000 charge/discharge cycles. The roughness of the paper fiber itself and the random network structure caused an uneven distribution of conductive materials, hindered electrolyte ion transport, and reduced electrochemical performance. Optimizing to obtain a uniform network structure and a reasonable pore structure of the paper electrode surface, it will be conducive to improving its electrochemical performance. In addition, a large amount of waste paper is generated in our daily life and work. If it is rationally designed and processed into paper-based electrodes, it not only can provide a way for the better development of flexible electrodes but also can realize waste reuse and protect the environment.

\subsection{Other types of flexible electrodes}

Except for the cellulose/CNT composite flexible electrode materials mentioned above, other forms of cellulose/CNT composite flexible electrode materials have also been used in supercapacitors. $\left.\begin{array}{lll}8 & 7\end{array}\right]$ The hierarchical CNFs/CNTs/PANI ternary composite electrode materials were fabricated via the electrospinning and in-situ polymerization methods ${ }^{[47]}$ (Fig. 10a). Benefiting from the abundant transmission channels of CNFs/CNTs, they can transmit and collect electrons more quickly, reduce the diffusion of electrolyte ions, and improve 


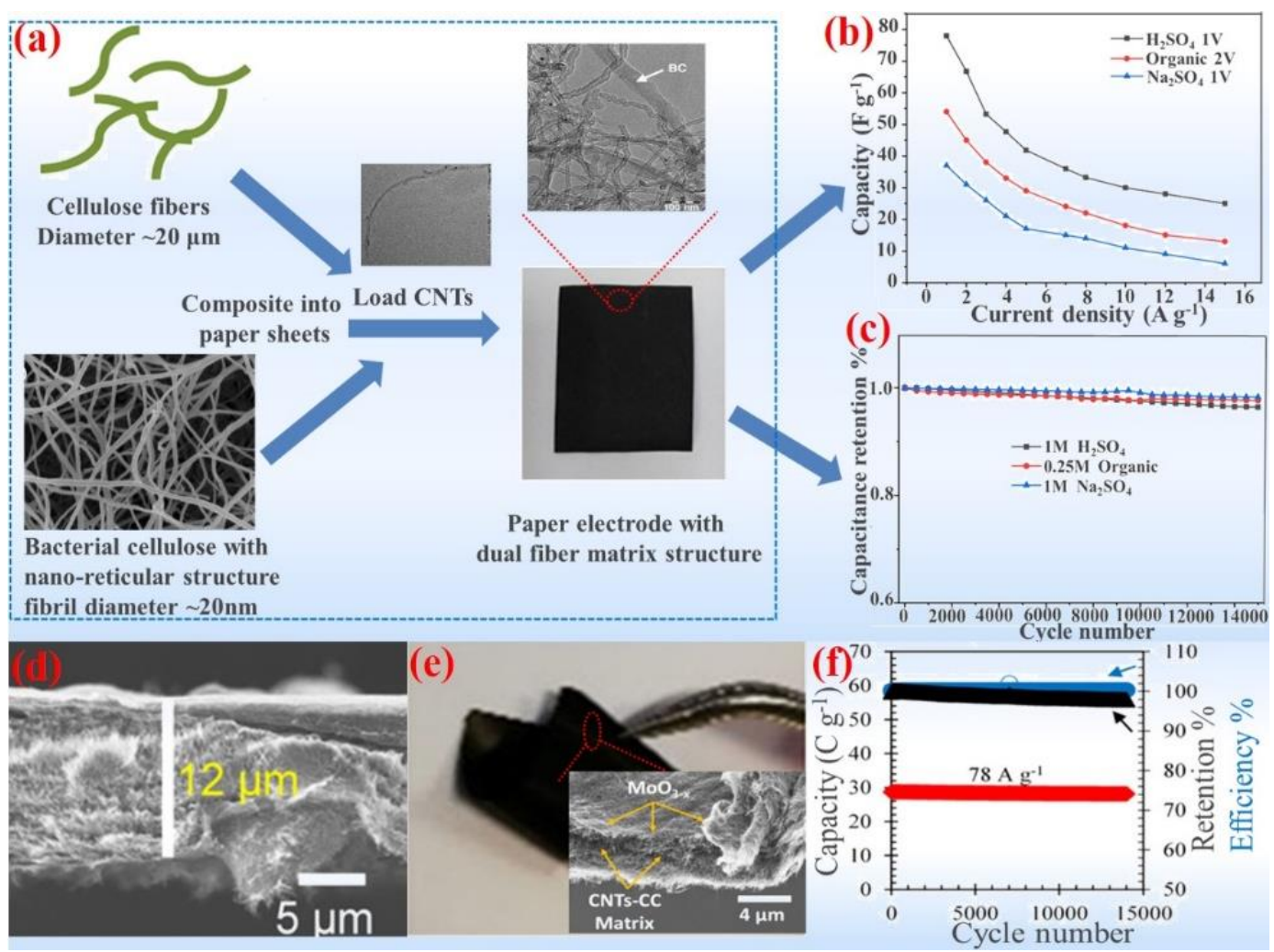

Fig. 9 (a) Schematic illustration of the paper electrode with a dual fiber matrix structure, (b) gravimetric capacitances at various currents, and (c) capacitance retention after 15,000 charge/discharge cycles in sulfuric acid, sodium sulfate, and organic electrolytes. Reproduced with the permission from [84] Copyright 2020 American Chemical Society; (d) SEM cross-section images, respectively, showing the thickness and homogeneity of the electrodes, (e) photos of the paper electrodes showing their flexibility (insets: SEM top-view image of $\mathrm{MoO}_{3-\mathrm{x}}$ on the CNTs-CC matrix), (f) discharge capacity (red), capacity retention (black), and coulombic efficiency (blue) as a function of the cycle number for current densities of $78 \mathrm{~A} \mathrm{~g}^{-1}$. Reproduced with permission from [85] Copyright 2019 Wiley-VCH Verlag GmbH \& Co. KGaA, Weinheim.

the utilization rate of pseudo-capacitance materials. The $\mathrm{CNF} / \mathrm{CNT} / \mathrm{PANI}$ electrode-specific capacitance can reach 315 $\mathrm{F} \mathrm{g}^{-1}$ at the current density of $1 \mathrm{~A} \mathrm{~g} \mathrm{~g}^{-1}$. The flexible solid supercapacitor has excellent flexibility, such as the capacitance maintained at $92 \%$ after 300 bends (Fig. 10b). In Fig. 10c, the electrochemical test results show that flexible supercapacitor has a large capacitance, good rate, high energy/power density (5.1 $\mathrm{Wh} \mathrm{kg}^{-1}$ at $\left.10.1 \mathrm{~kW} \mathrm{~kg}^{-1}\right)$, and excellent cycle stability (the capacitor retention rate is $92 \%$ after 10,000 cycles at the current density of $2 \mathrm{~A} \mathrm{~g}^{-1}$ ). The excellent electrochemical performance of the flexible electrode was contributed by the structural advantages of the material and the synergistic effect between the double capacitance and the pseudo-capacitance.

In the construction of flexible solid-state supercapacitors, the hierarchical pore structure was crucial to the improvement of the electrochemical performance. For example, Yao et al. ${ }^{[46]}$ prepared PPy@CNT/BC flexible electrodes with high electrochemical performance via self-assembly methods, and designed an all-solid-state flexible supercapacitor (FSC) with a layered core-sheath and porous structure. $\mathrm{BC}$ plays a dual role in FSC: 1) prevent the agglomeration of CNTs and improve the wettability of the electrode; 2) store the electrolyte as a nano-reservoir, which is beneficial to the diffusion of electrolyte ions. In addition, the layered coresheath and porous structure increase the specific surface area of the electrode, promote ion transport, and greatly improve its electrochemical performance. The capacitance of the PPy@CNT/BC electrode is $228 \mathrm{~F} \mathrm{~g}^{-1}$ at the current density of $0.5 \mathrm{~A} \mathrm{~g}^{-1}$. After 5000 cycles, the energy density of FSC can be maintained at $88 \%$ of the initial value. The electrochemical tests showed that FSC not only has the excellent bending ability and good cycle stability, but also has a large power density $\left(8.3 \mathrm{Wh} \mathrm{kg}^{-1}\right)$ and energy density

$\left(454.5 \mathrm{~W} \mathrm{~kg}^{-1}\right)$. Similarly, Wu et al..$^{[88]}$ used bacterial cellulose (BC), CNT, and polybenzazole (PIn) nanoparticles as supporting substrates, conductive channels, and electroactive materials, respectively. The PIn/CNT/BC flexible electrode with a layered structure was prepared by electrospinningelectrospray processes and constant potential polymerization methods. High conductivity and suitable charge transfer resistance make the $\mathrm{PIn} / \mathrm{CNT} / \mathrm{BC}$ flexible electrode have a larger capacitance $\left(552.6 \mathrm{~F} \mathrm{~g} \mathrm{~g}^{-1}\right)$, strong flexibility, and high cycle stability (capacitance retention rate after 5000 cycles was $95.6 \%)$.

Recently, metal-organic frameworks (MOFs) have been widely applied in the field of electrochemical energy storage as a new type of three-dimensional framework structure 

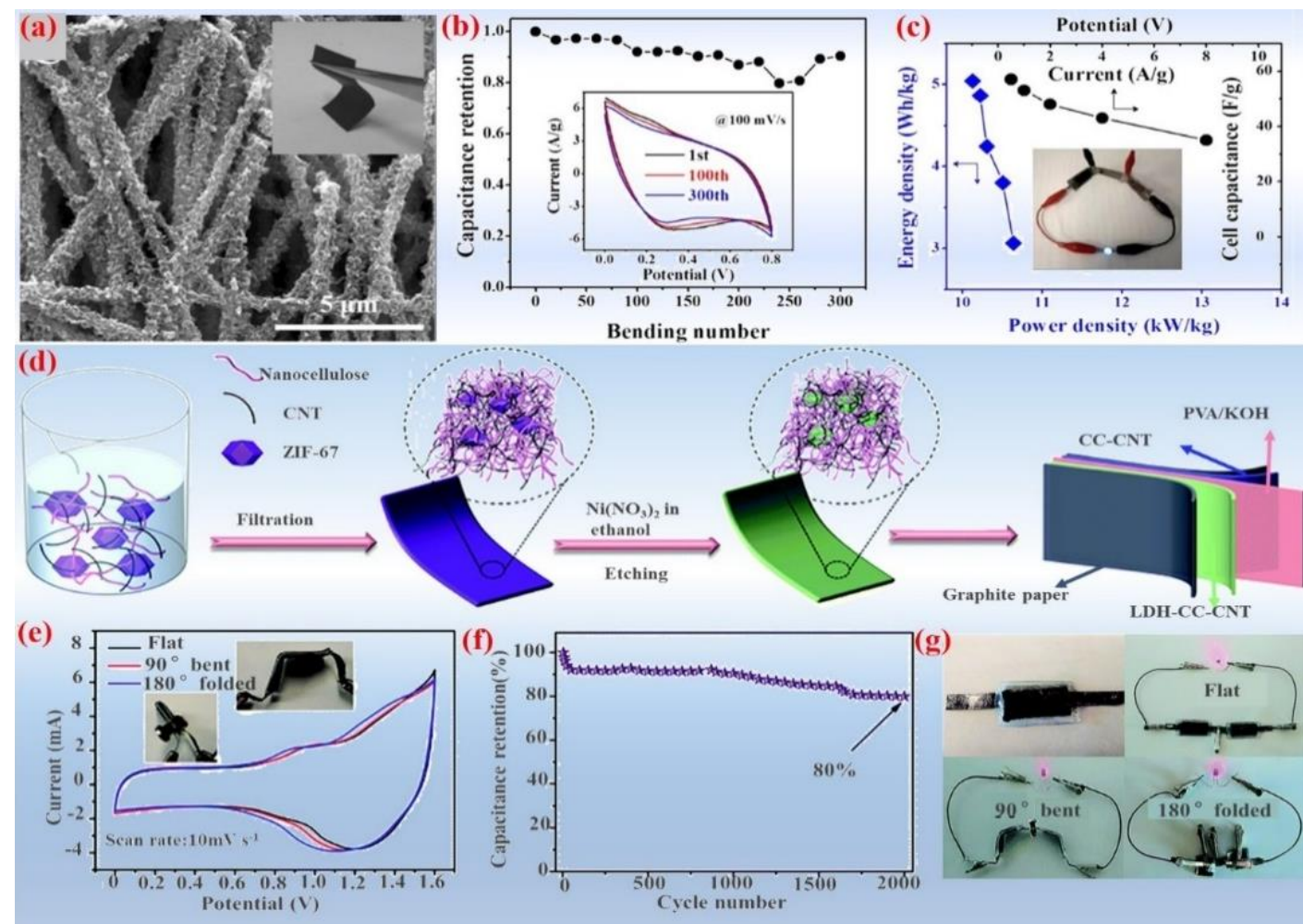

Fig. 10 (a) SEM images of CNFs/CNTs/PANI ternary composites, (b) Capacitance retention of the device after different bending numbers, (c) Ragone plot and cell capacitance of the device. Insert the application of the device. Reproduced with the permission from [47] Copyright 2016, American Chemical Society; (d) schematic illustration of the flexible and solid-state hybrid ESD LDH$\mathrm{CC}-\mathrm{CNT} / / \mathrm{CC}-\mathrm{CN}$, (e) $\mathrm{CV}$ curves of the device under different bending conditions, (f) cycling performance of the device, (g) photo of red LEDs powered by the bent or folded device. Reproduced with permission from [95] Copyright 2018, Royal Society of Chemistry.

materials. ${ }^{\left[{ }^{[9-94]}\right.}$ For example, Xu et al. ${ }^{[95]}$ fabricated a flexible foldable electrode (LDH-CC-CNT) by using dendritic cellulose (CC) nanofibers, CNTs, and layered Co-Ni double hydroxide (LDH) nanocages using ZIF-67 as a template. Subsequently, a flexible and foldable energy storage device (ESD) was constructed using LDH-CC-CNT and CC-CNT as the positive and negative electrodes and $\mathrm{PVA} / \mathrm{KOH}$ gel electrolyte (Fig. 10d). The test results of ESD showed that the surface capacitance was $168 \mathrm{mF} \mathrm{cm}^{-2}$, and the energy density reaches $60 \mu \mathrm{Wh} \mathrm{cm}^{-2}$ at the power density of $0.8 \mathrm{~mW} \mathrm{~cm}$. As shown in Fig. 10e, the CV curve remains almost unchanged in the case of $90^{\circ}$ and $180^{\circ}$ bending. After 2000 cycles, the ESD capacitance remains above $80 \%$ of the initial capacitance at the scanning rate of $50 \mathrm{mV} \mathrm{s}^{-1}$ (Fig. 10f).

The low capacitance retention rate was due to the deactivation of the metal during the rapid charge-discharge process and the reduction of the redox reaction. Fig. $10 \mathrm{~g}$ shows two connected hybrid ESD units in series that can still light up the LED lights after bending and folding. The use of MOFs and sustainably regenerated cellulose in flexible and foldable electronic energy storage devices have great potential. Electrochemical tests showed that the all-solid-state supercapacitor has good rate performance, high energy/power density, flexibility, and foldability, and provides a feasible strategy for the development of wearable and portable smart storage devices.

\section{Conclusion and Outlook}

Cellulose has widespread application prospects in the electrochemical flexible device field, owing to good biocompatibility, green sustainability, safety, non-toxicity, and excellent mechanical energy. The abundant pore structure of cellulose substrate provides a good conduction channel for electrolytes, which can accelerate the transport of electrolyte ions and improve electrochemical performance. Additionally, it can better disperse CNTs and prevent their agglomeration. Meanwhile, the unique internal structure, low mass density, and excellent electrical conductivity properties of CNT solve the non-conductive problem of cellulose.

In this review, we mainly introduce the preparation method of cellulose/CNT composites and the research progress of different forms of flexible electrodes for flexible supercapacitors. The shortcomings of cellulose/CNT composite materials are analyzed comprehensively and objectively, and reasonable improvement measures are proposed. With the increasing demand for smart, wearable, portable, and foldable devices, flexible supercapacitors have been deeply studied as indispensable components. From the perspective of environmental protection and the practicability of sustainable regeneration, it is a feasible strategy to optimize 
flexible electrode materials without impairing their excellent electrochemical performance. However, the cellulose/CNT composite flexible electrode material still has a competitive contradiction between charge storage, ion transport, and electronic conduction, which leads to the problems of low energy/power density and poor cycle stability in the application of supercapacitors. Therefore, the modification of composite materials, the optimization of pore structure, and the regulation of surface interface are still the main difficulties. To improve its overall performance to meet the actual application needs, the following four aspects are recommended for being optimized and improved:

(1) The selection of electrode materials directly affects the performance of supercapacitors. Therefore, it is important to select and prepare cellulose-based electrode materials. Firstly, the pore structure and porosity of cellulose and its composite materials should be optimized (such as grafting, doping, acidification, etc.), increasing the specific surface area of the composite material. Thus, it can have more active sites and better match the electrolyte ions.

(2) The interface between the electrode and the electrolyte needs to be properly addressed. The thickness of the diaphragm between the electrodes is as thin as possible, which can not only effectively reduce the distance between the electrodes and shorten the transmission path of electrons, but also increase the power density of the supercapacitor. Pretreatment of the surface of cellulose and CNT is the best way, which can improve the specific surface area and conductivity of composite electrode materials, and reduce the interface contact resistance of composite materials.

(3) Constructing green and efficient electronic devices is the goal of sustainable development. Therefore, it is necessary to seek environmentally friendly and scalable production of cellulose while maintaining the high-quality energy/power density and excellent cycle stability of cellulose-based supercapacitors.

(4) In order to further optimize the performance of the cellulose/CNT-based composite flexible electrode for supercapacitors. Employing a variety of methods is necessary for in-depth characterization and analysis to explore its reaction mechanism more accurately such as in-situ characterization and theoretical simulation calculations.

Cellulose-based composites not only be used as electrode materials for supercapacitors but also can be used as solid electrolyte and diaphragm components for supercapacitors and batteries. In future research, the above four aspects can serve as the research breakthrough points, and more precise experimental research should be adopted. Cellulose/CNTbased composite materials will be better utilized with their advantages in energy storage, sensing, biocompatibility, and environmental protection in engineering fields, such as energy storage, artificial skin, biomimetic devices, biomedicine, and aerospace.

\section{Acknowledgments}

This work was financially supported by the National Natural Science Foundation of China (No. 31670592), and the Natural Science Funds for Distinguished Young Scholar of Heilongjiang Province (No. JQ2019C001).

\section{Conflict of interest}

There are no conflicts to declare.

\section{Supporting information}

Applicable.

\section{References}

[1] H. Chen, T. Liu, J. Mou, W. Zhang, Z. Jiang, J. Liu, J. Huang, M. Liu, Nano Energy, 2019, 63, 103836, doi: 10.1016/j.nanoen.2019.06.032.

[2] L. Xia, X. Li, X. Wu, L. Huang, Y. Liao, Y. Qing, Y. Wu, X. Lu, Journal of Materials Chemistry A, 2018, 6, 17378-17388, doi: 10.1039/c8ta05678a.

[3] H. Zhang, Z. Zhang, X. Qi, J. Yu, J. Cai, Z. Yang, ACS Sustainable Chemistry \& Engineering, 2019, 7, 4284-4294, doi: 10.1021/acssuschemeng.8b06049.

[4] Q. Zhang, C. Chen, W. Chen, G. Pastel, X. Guo, S. Liu, Q. Wang, Y. Liu, J. Li, H. Yu, L. Hu, ACS Applied Materials \& Interfaces, 2019, 11, 5919-5927, doi: 10.1021/acsami.8b17414.

[5] P. E. Lokhande, U. S. Chavan, A. Pandey, Electrochemical Energy Reviews, 2020, 3, 155-186, doi: 10.1007/s41918-01900057-z.

[6] Y. Wang, Z. Zhao, W. Song, Z. Wang, X. Wu, Journal of Materials Science, 2019, 54, 4917-4927, doi: 10.1007/s10853018-03215-8.

[7] T. Lv, M. Liu, D. Zhu, L. Gan, T. Chen, Advanced Materials, 2018, 30, e1705489-e1705505, doi: 10.1002/adma.201705489.

[8] R. J. Moon, A. Martini, J. Nairn, J. Simonsen, J. Youngblood, Chemical Society Reviews, 2011, 40, 3941, doi: 10.1039/c0cs00108b.

[9] Z. Wang, Y. Tan, Y. Yang, X. Zhao, Y. Liu, L. Niu, B. Tichnell, L. Kong, L. Kang, Z. Liu, F. Ran, Journal of Power Sources, 2018 , 378, 499-510, doi: 10.1016/j.jpowsour.2017.12.076.

[10] N. P. Marinho, P. H. G. de Cademartori, S. Nisgoski, V. O. de Andrade Tanobe, U. Klock, G. I. B. de Muñiz, Carbohydrate Polymers, 2020, 230, 115579, doi: 10.1016/j.carbpol.2019.115579.

[11] T. I. Shaheen, H. E. Emam, International journal of biological macromolecules, 2018, 107, 1599-1606, doi: 10.1016/j.ijbiomac.2017.10.028.

[12] S. Li, D. Huang, B. Zhang, X. Xu, M. Wang, G. Yang, Y. Shen, Advanced Energy Materials, 2014, 4, 1301655, doi: 10.1002/aenm.201301655.

[13] F. L. Dri, L. G. Hector Jr, R. J. Moon, P. D. Zavattieri, Cellulose, 2013, 20, 2703-2718, doi: 10.1007/s10570-013-00718.

[14] L. Ma, R. Liu, H. Niu, M. Zhao, Y. Huang, Composites Science and Technology, 2016, 137, 87-93, doi: 10.1016/j.compscitech.2016.10.027. 
[15] U. Henniges, S. Veigel, E. M. Lems, A. Bauer, J. Keckes, S. Pinkl, W. Gindl-Altmutter, Cellulose, 2014, 21, 1601-1610, doi: 10.1007/s10570-014-0232-4.

[16] P. Gatenholm, D. Klemm, MRS Bulletin, 2010, 35, 208-213, doi: $10.1557 / \mathrm{mrs} 2010.653$.

[17] Z. Sun, K. Qu, Y. You, Z. Huang, S. Liu, J. Li, Q. Hu, Z. Guo, Journal of Materials Chemistry A, 2021, 9, 7278-7300, doi: 10.1039/D0TA10504J.

[18] H. Song, S. Xu, Y. Li, J. Dai, A. Gong, M. Zhu, C. Zhu, C. Chen, Y. Chen, Y. Yao, B. Liu, J. Song, G. Pastel, L. Hu, Advanced Energy Materials, 2018, 8, 1701203, doi: 10.1002/aenm.201701203.

[19] J. Jose, V. Thomas, V. Vinod, R. Abraham, S. Abraham, Journal of Science: Advanced Materials and Devices, 2019, 4, 333-340, doi: 10.1016/j.jsamd.2019.06.003.

[20] S. He, C. Zhang, C. Du, C. Cheng, W. Chen, Journal of Power Sources, 2019, 434, 226701, doi: 10.1016/j.jpowsour.2019.226701.

[21] Y. M. Fan, W. L. Song, X. Li, L. Z. Fan, Carbon, 2017, 111, 658-666, doi: 10.1016/j.carbon.2016.10.056.

[22] X. L. Zhang, H. J. Fu, G. R. Yang, G. Wan, D. Li, L. X. Zhu, R. R. Xie, Y. J. Lv, J. D. Zhang, Y. L. Li, Q. F. Dai, Y. Ji, D. Y. Gao, X. L. Cui, D. Y. Liu, S. Y. Yuan, M. X. Yuan, Diabetes Research and Clinical Practice, 2018, 144, 236-244, doi: 10.1016/j.diabres.2018.09.007.

[23] H. Li, D. Yuan, C. Tang, S. Wang, J. Sun, Z. Li, T. Tang, F. Wang, H. Gong, C. He, Carbon, 2016, 100, 151-157, doi: 10.1016/j.carbon.2015.12.075.

[24] X. Li, J. Zhao, Z. Cai, F. Ge, Materials Research Bulletin, 2018, 107, 355-360, doi: 10.1016/j.materresbull.2018.08.012.

[25] P. Schlee, O. Hosseinaei, D. Baker, A. Landmér, P. Tomani, M. J. Mostazo-López, D. Cazorla-Amorós, S. Herou, M. M. Titirici, Carbon, 2019, 145, 470-480, doi: 10.1016/j.carbon.2019.01.035.

[26] Z. Tang, Z. Pei, Z. Wang, H. Li, J. Zeng, Z. Ruan, Y. Huang, M. Zhu, Q. Xue, J. Yu, C. Zhi, Carbon, 2018, 130, 532-543, doi: 10.1016/j.carbon.2018.01.055.

[27] X. Hao, J. Wang, B. Ding, Y. Wang, Z. Chang, H. Dou, X. Zhang, Journal of Power Sources, 2017, 352, 34-41, doi: 10.1016/j.jpowsour.2017.03.088.

[28] S. Hu, S. Zhang, N. Pan, Y. L. Hsieh, Journal of Power Sources, 2014, 270, 106-112, doi: 10.1016/j.jpowsour.2014.07.063.

[29] C. Lai, Z. Zhou, L. Zhang, X. Wang, Q. Zhou, Y. Zhao, Y. Wang, X. F. Wu, Z. Zhu, H. Fong, Journal of Power Sources, 2014, 247, 134-141, doi: 10.1016/j.jpowsour.2013.08.082.

[30] Z. Zhang, L. Li, Y. Qing, X. Lu, Y. Wu, N. Yan, W. Yang, The Journal of Physical Chemistry C, 2019, 123, 23374-23381, doi: 10.1021/acs.jpcc.9b06058.

[31] J. Han, S. Wang, S. Zhu, C. Huang, Y. Yue, C. Mei, X. Xu, C. Xia, ACS Applied Materials \& Interfaces, 2019, 11, 4462444635, doi: 10.1021/acsami.9b16458.

[32] A. Divyashree, G. Hegde, RSC Advances, 2015, 5, 8833988352, doi: 10.1039/c5ra19392c.

[33] Q. Zheng, Z. Cai, Z. Ma, S. Gong, ACS Applied Materials \&
Interfaces, 2015, 7, 3263-3271, doi: 10.1021/am507999s.

[34] Y. Lv, Y. Zhou, Z. Shao, Y. Liu, J. Wei, Z. Ye, Journal of Materials Science: Materials in Electronics, 2019, 30, 8585-8594, doi: 10.1007/s10854-019-01180-9.

[35] L. Ma, R. Liu, H. Niu, L. Xing, L. Liu, Y. Huang, ACS Applied Materials \& Interfaces, 2016, 8, 33608-33618, doi: 10.1021/acsami.6b11034.

[36] D. Ge, L. Yang, L. Fan, C. Zhang, X. Xiao, Y. Gogotsi, S. Yang, Nano Energy, 2015, 11, 568-578, doi: 10.1016/j.nanoen.2014.11.023.

[37] L. Ma, R. Liu, L. Liu, F. Wang, H. Niu, Y. Huang, Journal of Power Sources, 2016, 335, 76-83, doi: 10.1016/j.jpowsour.2016.10.006.

[38] H. Luo, J. Dong, Y. Zhang, G. Li, R. Guo, G. Zuo, M. Ye, Z. Wang, Z. Yang, Y. Wan, Chemical Engineering Journal, 2018, 334, 1148-1158, doi: 10.1016/j.cej.2017.11.065.

[39] D. Zhao, Y. Zhu, W. Cheng, W. Chen, Y. Wu, H. Yu, Advanced Materials, 2021, 33, 2000619, doi: 10.1002/adma.202000619.

[40] Z. Fan, J. Yan, L. Zhi, Q. Zhang, T. Wei, J. Feng, M. Zhang, W. Qian, F. Wei, Advanced Materials, 2010, 22, 3723-3728, doi: 10.1002/adma.201001029.

[41] Z. Tang, C. H. Tang, H. Gong, Advanced Functional Materials, 2012, 22, 1272-1278, doi: 10.1002/adfm.201102796. [42] F. H. Kuok, K. Y. Kan, I. S. Yu, C. W. Chen, C. C. Hsu, I. C. Cheng, J. Z. Chen, Applied Surface Science, 2017, 425, 321-328, doi: 10.1016/j.apsusc.2017.06.286.

[43] F.-H. Kuok, C.-Y. Liao, C.-W. Chen, Y.-C. Hao, I.-S. Yu, J.Z. Chen, Materials Research Express, 2017, 4, 115501, doi: 10.1088/2053-1591/aa9405.

[44] K. Gao, Z. Shao, X. Wang, Y. Zhang, W. Wang, F. Wang, RSC Advances, 2013, 3, 15058, doi: 10.1039/c3ra42050g.

[45] L. Xia, X. Li, Y. Wu, S. Hu, Y. Liao, L. Huang, Y. Qing, X. Lu, Chemical Engineering Journal, 2020, 379, 122325, doi: 10.1016/j.cej.2019.122325.

[46] J. Yao, P. Ji, N. Sheng, F. Guan, M. Zhang, B. Wang, S. Chen, H. Wang, Electrochimica Acta, 2018, 283, 1578-1588, doi: 10.1016/j.electacta.2018.07.086.

[47] F. Miao, C. Shao, X. Li, K. Wang, N. Lu, Y. Liu, ACS Sustainable Chemistry \& Engineering, 2016, 4, 1689-1696, doi: 10.1021/acssuschemeng.5b01631.

[48] Q. Zhao, J. Liu, X. Li, Z. Xia, Q. Zhang, M. Zhou, W. Tian, M. Wang, H. Hu, Z. Li, W. Wu, H. Ning, M. Wu, Chemical Engineering Journal, 2019, 369, 215-222, doi: 10.1016/j.cej.2019.03.076.

[49] Y. Bai, R. Liu, E. Li, X. Li, Y. Liu, G. Yuan, Journal of Alloys and Compounds, 2019, 777, 524-530, doi: 10.1016/j.jallcom.2018.10.376.

[50] B. Zhang, J. Zhou, S. Li, X. Zhang, D. Huang, Y. He, M. Wang, G. Yang, Y. Shen, Talanta, 2015, 131, 243-248, doi: 10.1016/j.talanta.2014.07.027.

[51] W. Chen, H. Yu, S.-Y. Lee, T. Wei, J. Li, Z. Fan, Chemical Society Reviews, 2018, 47, 2837-2872, doi: 10.1039/c7cs00790f. [52] S. Xie, X. Zhang, M. P. Walcott, H. Lin, Engineered Science, 2018, 2, 4-16, doi: 10.30919/es.1803302. 
[53] H. H. Hsu, W. Zhong, Membranes, 2019, 9, 74, doi: 10.3390/membranes9060074.

[54] Z. Chen, T. Yan, Z. Pan, Cellulose, 2021, 28, 615-645, doi: 10.1007/s10570-020-03543-6.

[55] S. Dutta, J. Kim, Y. Ide, J. Ho Kim, M. S. A. Hossain, Y. Bando, Y. Yamauchi, K. C. W. Wu, Materials Horizons, 2017, 4, 522-545, doi: 10.1039/c6mh00500d.

[56] Z. Wang, Y. H. Lee, S. W. Kim, J. Y. Seo, S. Y. Lee, L. Nyholm, Advanced Materials, 2021, 33, 2000892, doi: 10.1002/adma.202000892.

[57] S. Xu, W. Yu, M. Jing, R. Huang, Q. Zhang, Q. Fu, The Journal of Physical Chemistry C, 2017, 121, 2108-2117, doi: 10.1021/acs.jpcc.6b11783.

[58] M. Wang, I. V. Anoshkin, A. G. Nasibulin, R. H. A. Ras, N. Nonappa, J. Laine, E. I. Kauppinen, O. Ikkala, RSC Advances, 2016, 6, 89051-89056, doi: 10.1039/c6ra16202a.

[59] P. Zhu, Y. Liu, Z. Fang, Y. Kuang, Y. Zhang, C. Peng, G. Chen, Langmuir, 2019, 35, 4834-4842, doi: 10.1021/acs.langmuir.8b04259.

[60] A. W. Jatoi, H. Ogasawara, I. S. Kim, Q.-Q. Ni, Materials Science and Engineering: C, 2020, 110, 110679, doi: 10.1016/j.msec.2020.110679.

[61] M. Miyauchi, J. Miao, T. J. Simmons, J. W. Lee, T. V. Doherty, J. S. Dordick, R. J. Linhardt, Biomacromolecules, 2010, 11, 2440-2445, doi: 10.1021/bm1006129.

[62] H. Qi, B. Schulz, T. Vad, J. Liu, E. Mäder, G. Seide, T. Gries, ACS Applied Materials \& Interfaces, 2015, 7, 22404-22412, doi: 10.1021/acsami.5b06229.

[63] S. Y. Cho, H. Yu, J. Choi, H. Kang, S. Park, J. S. Jang, H. J. Hong, I. D. Kim, S. K. Lee, H. S. Jeong, H. T. Jung, ACS Nano, 2019, 13, 9332-9341, doi: 10.1021/acsnano.9b03971.

[64] L. Deng, R. J. Young, I. A. Kinloch, A. M. Abdelkader, S. M. Holmes, D. A. de Haro-Del Rio, S. J. Eichhorn, ACS Applied Materials \& Interfaces, 2013, 5, 9983-9990, doi: 10.1021/am403622v.

[65] Y. Liu, Y. Wang, Y. Nie, C. Wang, X. Ji, L. Zhou, F. Pan, S. Zhang, ACS Sustainable Chemistry \& Engineering, 2019, 7, 20013-20021, doi: 10.1021/acssuschemeng.9b05489.

[66] D. Zhao, Q. Zhang, W. Chen, X. Yi, S. Liu, Q. Wang, Y. Liu, J. Li, X. Li, H. Yu, ACS Applied Materials \& Interfaces, 2017, 9 , 13213-13222, doi: 10.1021/acsami.7b01852.

[67] A. M. R. Rebelo, Y. Liu, C. Liu, K.-H. Schäfer, M. Saumer, G. Yang, ACS Biomaterials Science \& Engineering, 2019, 5, 2160-2172, doi: 10.1021/acsbiomaterials.9b00039.

[68] J. H. Mo, J. Y. Kim, Y. H. Kang, S. Y. Cho, K. S. Jang, ACS Sustainable Chemistry \& Engineering, 2018, 6, 15970-15975, doi: 10.1021/acssuschemeng.8b03670.

[69] H. Zhang, X. Sun, Z. Heng, Y. Chen, H. Zou, M. Liang, Industrial \& Engineering Chemistry Research, 2018, 57, $17152-$ 17160, doi: 10.1021/acs.iecr.8b04573.

[70] C. Avendano, N. Brun, O. Fontaine, M. In, A. Mehdi, A. Stocco, A. Vioux, Langmuir, 2016, 32, 3907-3916, doi: 10.1021/acs.langmuir.6b00380.

[71] H. Wang, E. Zhu, J. Yang, P. Zhou, D. Sun, W. Tang, The Journal of Physical Chemistry C, 2012, 116, 13013-13019, doi: 10.1021/jp301099r.

[72] C. Chen, M. Mo, W. Chen, M. Pan, Z. Xu, H. Wang, D. Li, Composites Science and Technology, 2018, 156, 103-108, doi: 10.1016/j.compscitech.2017.12.029.

[73] K. Qu, Z. Sun, C. Shi, W. Wang, L. Xiao, J. Tian, Z. Huang, Z. Guo, Advanced Composites and Hybrid Materials, 2021, 4, 670-683, doi: 10.1007/s42114-021-00293-2.

[74] S. Lyu, Y. Chen, L. Zhang, S. Han, Y. Lu, Y. Chen, N. Yang, Z. Chen, S. Wang, RSC Advances, 2019, 9, 17824-17834, doi: 10.1039/c9ra02449b.

[75] L.-Q. Zhang, S.-G. Yang, L. Li, B. Yang, H.-D. Huang, D.X. Yan, G.-J. Zhong, L. Xu, Z.-M. Li, ACS Applied Materials \& Interfaces, 2018, 10, 40156-40167, doi: 10.1021/acsami.8b14738. [76] H. Xu, X. Yin, M. Li, X. Li, X. Li, X. Dang, L. Zhang, L. Cheng, ACS Applied Materials \& Interfaces, 2019, 11, 2262822636, doi: 10.1021/acsami.9b03731.

[77] Y. Bu, M. Cao, Y. Jiang, L. Gao, Z. Shi, X. Xiao, M. Wang, G. Yang, Y. Zhou, Y. Shen, Electrochimica Acta, 2018, 271, 624631, doi: 10.1016/j.electacta.2018.03.155.

[78] J. Cai, H. Niu, Z. Li, Y. Du, P. Cizek, Z. Xie, H. Xiong, T. Lin, ACS Applied Materials \& Interfaces, 2015, 7, 14946-14953, doi: 10.1021/acsami.5b03757.

[79] J. Han, H. Wang, Y. Yue, C. Mei, J. Chen, C. Huang, Q. Wu, X. Xu, Carbon, 2019, 149, 1-18, doi: 10.1016/j.carbon.2019.04.029.

[80] X. Shi, Y. Hu, M. Li, Y. Y. Duan, Y. Wang, L. Chen, L. Zhang, Cellulose, 2014, 21, 2337-2347, doi: 10.1007/s10570-014-02881.

[81] H. D. Huang, C. Y. Liu, L. Q. Zhang, G. J. Zhong, Z. M. Li, ACS Sustainable Chemistry \& Engineering, 2015, 3, 317-324, doi: $10.1021 / \mathrm{sc} 500681 \mathrm{v}$

[82] J. P. Jyothibasu, D. W. Kuo, R. H. Lee, Cellulose, 2019, 26, 4495-4513, doi: 10.1007/s10570-019-02376-2.

[83] D. Müller, J. S. Mandelli, J. A. Marins, B. G. Soares, L. M. Porto, C. R. Rambo, G. M. O. Barra, Cellulose, 2012, 19, $1645-$ 1654, doi: 10.1007/s10570-012-9754-9.

[84] X. Wu, M. Zhang, T. Song, H. Mou, Z. Xiang, H. Qi, ACS Applied Materials \& Interfaces, 2020, 12, 13096-13106, doi: 10.1021/acsami.9b19347.

[85] A. S. Etman, Z. Wang, A. El Ghazaly, J. Sun, L. Nyholm, J. Rosen, ChemSusChem, 2019, 12, 5157-5163, doi: 10.1002/cssc. 201902394.

[86] B. S. Noremberg, R. M. Silva, O. G. Paniz, J. H. Alano, J. Dupont, N. L. V. Carreño, MRS Communications, 2019, 9, 726729, doi: $10.1557 / \mathrm{mrc} .2019 .34$.

[87] J.-W. Han, B. Kim, J. Li, M. Meyyappan, The Journal of Physical Chemistry C, 2012, 116, 22094-22097, doi: 10.1021/jp3080223.

[88] J. Wu, Z. Du, P. Xiong, Z. Cai, Cellulose, 2020, 27, 6353 6366, doi: 10.1007/s10570-020-03199-2.

[89] L. Xiao, H. Qi, K. Qu, C. Shi, Y. Cheng, Z. Sun, B. Yuan, Z. Huang, D. Pan, Z. Guo, Advanced Composites and Hybrid Materials, 2021, 4, 306-316, doi: 10.1007/s42114-021-00223-2.

[90] S. Zhou, X. Kong, B. Zheng, F. Huo, M. Strømme, C. Xu, ACS Nano, 2019, 13, 9578-9586, doi: 10.1021/acsnano.9b04670. 
[91] J. Zhou, Y. Yuan, J. Tang, W. Tang, Energy Storage Materials, 2019, 23, 594-601, doi: 10.1016/j.ensm.2019.03.024. [92] Y.-N. Liu, H.-T. Wang, X.-H. Kang, Y.-F. Wang, S.-Y. Yang, S.-W. Bian, Journal of Power Sources, 2018, 402, 413-421, doi: 10.1016/j.jpowsour.2018.09.052.

[93] Z. Li, L. Yang, H. Cao, Y. Chang, K. Tang, Z. Cao, J. Chang, Y. Cao, W. Wang, M. Gao, C. Liu, D. Liu, H. Zhao, Y. Zhang, M. Li, Carbohydrate Polymers, 2017, 175, 223-230, doi: 10.1016/j.carbpol.2017.07.089.

[94] J. Cai, W. Xu, Y. Liu, Z. Zhu, G. Liu, W. Ding, G. Wang, H. Wang, Y. Luo, Engineered Science, 2019, 5, 21-29 doi: 10.30919/es8d669.

[95] C. Xu, X. Kong, S. Zhou, B. Zheng, F. Huo, M. Strømme, Journal of Materials Chemistry A, 2018, 6, 24050-24057, doi: 10.1039/C8TA10133G.

\section{Authors information}

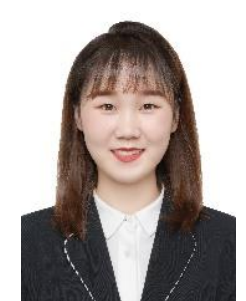

Zhe Sun is currently a third year Ph. D. student in Material Science and Engineering College, Northeast Forestry University. She received her B.E. degree in chemical engineering and technology from Heze University in 2017. Her current research includes the application of biomass carbon and metal organic framework composite materials in catalysis, adsorption and supercapacitors.

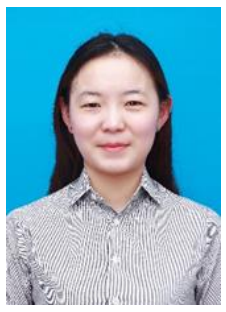

Houjuan Qi studied in Northeast Forestry University (M.S. and Ph.D., 2021), China. She mainly focuses on biomass-based heterogeneous composite materials and biomass-based slow-release materials.

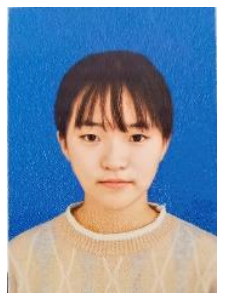

Manhui Chen is currently a third-year undergraduate student in Forestry Engineering College, Northeast Forestry University. Her current research is the preparation and functional application of multi-responsive sodium alginate composite hydrogel.

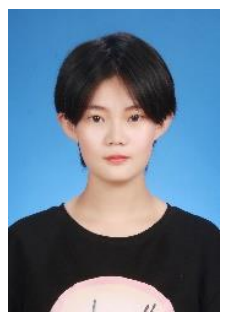

Sitong Guo is currently a third-year undergraduate student in Forestry Engineering College, Northeast Forestry University. Her current research is the application of cellulose and carbon materials for electrode materials.

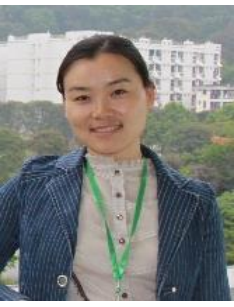

Zhanhua Huang is a professor of the Key laboratory of Bio-based Material Science and Technology of Ministry of Education at Northeast Forestry University, China. She received her B.E. in chemical from Northeast Forestry University in 1999, and her M.S. and Ph.D. in forest products chemical processing from Northeast Forestry University in 2003 and 2006, respectively. She was appointed assistant professor of Northeast Forestry University in 2006, associate professor in 2009, and professor in 2014. Her research interests include functional bio-material adsorbents, green conversion of sustainable and renewable biomass, novel biomass-based photocatalytic materials and cellulose-based flexible electronics.

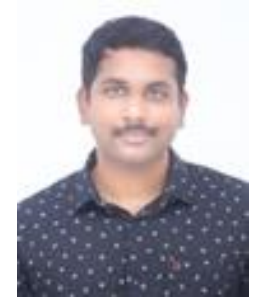

Vignesh Murugadoss, obtained his Ph.D. from Centre for nanoscience and Technology at Pondicherry University, Puducherry. He carried out Indo-US BASE internship at Integrated Composites Laboratory, Department of Chemical and Biomolecular Engineering at University of Tennessee, Knoxville. His research interests focus on design and development of advanced functional materials for sustainable energy and environmental applications.

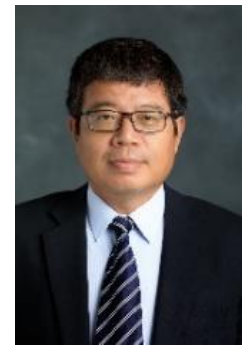

Zhanhu Guo, an Associate Professor in the Department of Chemical and Biomolecular Engineering, University of Tennessee, Knoxville, USA. He obtained a Ph.D. degree in Chemical Engineering from Louisiana State University (2005), and then received three-year (2005-2008) postdoctoral training from the Mechanical and Aerospace Engineering Department at the University of California Los Angeles. Dr Guo chaired the Composite Division of the American Institute of Chemical Engineers (AIChE, 2010-2011). Dr. Guo is the Lifetime Fellow of Indian Chemical Society (ICS, 2020) and Fellow of the Institute of Materials, Minerals and Mining (FIMMM, 2021). Dr. Guo is the director of the Integrated Composites Laboratory. His current research focuses on multifunctional nanocomposites for energy, electronic and environmental applications.

Publisher's Note: Engineered Science Publisher remains neutral with regard to jurisdictional claims in published maps and institutional affiliations. 